\title{
Control strategies used in lower limb exoskeletons for gait rehabilitation after brain injury: a systematic review and analysis of clinical effectiveness
}

Jesus de Miguel-Fernandez

Universitat Politecnica de Cataluna Centre de Recerca en Enginyeria Biomedica https://orcid.org/00000001-8651-1642

Joan Lobo-Prat

ABLE Human Motion

\section{Erik Prinsen}

Roessingh Research and Development

\section{Josep Maria Font-Llagunes}

Universitat Politècnica de Catalunya: Universitat Politecnica de Catalunya

Laura Marchal-Crespo ( $\square$ laura.marchal@unibe.ch )

University of Bern: Universitat Bern https://orcid.org/0000-0002-8008-5803

\section{Research Article}

Keywords: Powered Exoskeleton, Gait Rehabilitation, Lower-limb, Brain Injury, Stroke, Cerebral Palsy, Literature synthesis

Posted Date: January 28th, 2022

DOI: https://doi.org/10.21203/rs.3.rs-1195778/v1

License: (9) This work is licensed under a Creative Commons Attribution 4.0 International License. Read Full License 


\title{
Control strategies used in lower limb exoskeletons for gait rehabilitation after brain injury: a systematic review and analysis of clinical effectiveness
}

\author{
Jesús de Miguel-Fernández ${ }^{1,2}$, Joan Lobo-Prat ${ }^{3}$, Erik Prinsen ${ }^{4}$, Josep M. Font-Llagunes ${ }^{1,2,3}$ and Laura \\ Marchal-Crespo ${ }^{5,6^{*}}$
}

\begin{abstract}
Background: In the past decade, there has been substantial progress in the development of robotic controllers that specify how lower-limb exoskeletons should interact with brain-injured patients. However, it is still an open question which exoskeleton control strategies can more effectively stimulate motor function recovery. Within this review, we aim to complement previous literature surveys on the topic of exoskeleton control for gait rehabilitation by: (1) Providing an updated structured framework of current control strategies, (2) Analyzing the methodology of clinical validations used in the robotic interventions, and (3) Reporting the potential relation between the employed control strategies and clinical outcomes.
\end{abstract}

Methods: Four databases were searched using database-specific search terms from 2000 to September 2020. We identified 1648 articles, of which 159 were included and evaluated in full-text. We included studies that clinically evaluated the effectiveness of the exoskeleton on impaired participants, and which clearly explained or referenced the implemented control strategy.

Results: (1) We found that adaptive assistive control (100\% of exoskeletons) that followed rule-based algorithms $(72 \%)$ based on ground reaction force thresholds $(63 \%)$ in conjunction with trajectory-tracking control $(97 \%)$ were the most implemented control strategies. (2) Regarding the clinical validations used in the robotic interventions, we found high variability on the experimental protocols and outcome metrics selected. (3) With moderate grade of evidence, associated to the high heterogeneity in the experimental protocol and low number of studies, we found that adaptive control strategies, which followed threshold-based or adaptive oscillator algorithms together with trajectory-tracking control, resulted in the highest improvements on clinical outcomes for people with stroke.

Conclusions: Despite the efforts to develop novel more effective controllers for gait neurorehabilitation, the current level of evidence on the effectiveness of the different control strategies on clinical outcomes is still low. There is a clear lack of standardization in the experimental protocols leading to high levels of heterogeneity. Standardized comparisons among control strategies analyzing the relation between control parameters and biomechanical metrics will fill this gap to better guide future technical developments. The most promising controllers seem to be those that adapt to key biomechanical descriptors based on the patients' specific pathology.

Keywords: Powered Exoskeleton; Gait Rehabilitation; Lower-limb; Brain Injury; Stroke; Cerebral Palsy; Literature synthesis

\footnotetext{
${ }^{*}$ Correspondence: laura.marchal@unibe.ch

${ }^{6}$ Motor Learning and Neurorehabilitation Lab, ARTORG Center for Biomedical Engineering Research, University of Bern, Freiburgstrasse 3, 3010, Bern, Switzerland

Full list of author information is available at the end of the article
}

\section{Background}

Brain injuries, e.g., stroke, cerebral palsy $(\mathrm{CP})$, and traumatic brain injury, are one of the major causes of death and disability worldwide [1]. The global incidence of stroke increases by more than 13.7 million 
6 new cases each year [2], and is the third leading cause of disability worldwide [3]. The prevalence of cerebral palsy is estimated to be from nearly 2 to nearly 3 per 1,000 newborns worldwide $[4,5]$. Traumatic brain injury is another leading cause of disability around the globe, with 69 million survivors every year [6].

Difficulty standing and walking is one of the major consequences of brain injuries. For instance, over 63 $\%$ of stroke survivors suffer from half-mild to severe motor and cognitive disabilities [7], and 30-36 \% are unable to walk without assistive aids $[8,9]$. This results in loss of independent mobility and limits community participation and social integration, which causes secondary health conditions [10]. People with different brain injuries can exhibit common motor impairments, like paralysis, spasticity, or abnormal muscle synergies, leading to compensatory movements and gait asymmetries [11-15]. This pathological gait hinders a skilful, comfortable, safe, and metabolically efficient ambulation [16].

The recovery process after a brain injury takes months to years and neurological impairments can be permanent [17]. There is strong evidence that early, intensive, and repetitive task- and goal-oriented training, which is progressively adapted to the patient's level of impairment and rehabilitation stage, can improve functional ambulatory outcomes [11,18-23]. However, due to limited resources and the heterogeneity of impairment, it is challenging for physiotherapists to provide the required intensity and dose of training, while extracting quantitative information to maximize functional walking ability for a specific patient.

Robotics can play a promising role in gait rehabilitation for people with brain injuries. Robots allow performance of wide range of tasks -e.g., walking, sitting up/down, or walking on a slope- with high intensity. Some robotic controllers might also promote patients' active participation and engagement during the training process, e.g., by varying the level of the assistive force $[24,25]$. High repeatability and intensity of training, together with patients' engagement, have been listed as crucial factors to induce neural plasticity and motor learning [26-28]. Importantly, clinical evidence suggests that combining robotic and conventional rehabilitation training positively impacts the ability to walk independently, walking speed, and walking capacity, although there is still no solid evidence about the superiority of robotic rehabilitation over conventional therapy [29-33].

Lower-limb exoskeletons promote task-oriented repetitive movements, muscle strengthening, and movement coordination, which have shown to positively impact energy efficiency, gait speed, and balance control $[34,35]$. Exoskeletons, compared to other robotic solutions, e.g., patient-guided suspension systems and end-effector devices, allow for full control of the leg joint angles and torques, and are the preferred solutions for training brain-injured patients who suffer from severe disabilities [36]. Thereby, we consider that focusing on exoskeleton technology is a wide and rich enough topic to extract conclusions on the clinical effectiveness of the control strategies in the broad group of brain-injured patients [37-39].

The interest on lower-limb exoskeletons for gait rehabilitation has increased exponentially in the last years, which is reflected in the considerable number of reviews published within the last decade $[38,40-60]$. However, the majority of these reviews focus on hardware, while only a few of them analyzed the control strategies implemented on lower limb exoskeletons and their effects on walking function in people with brain injuries $[38,41,42,54-60]$. Yet, the control strategy as ergonomics and robot actuation- might play a key role on the effectiveness of the robotic treatment [61]. As in every biological system, control rules are essential to modulate every action attending to internal and external factors [62].

We found a few literature surveys that focused on control strategies for lower-extremity exoskeletons: Baud et al. and Li et al. categorised the control strategies and actuation systems implemented on lower-limb exoskeletons [41,42]; Chen et al. presented a review on wearable hip exoskeletons for gait rehabilitation and human performance augmentation that addressed actuation system technologies and control strategies [57]; Zhang et al. presented a review on lower-limb exoskeletons offering details about actuation systems, high-level control, and human-robot synchronization tools [38]; Tucker et al. [55] reviewed several control strategies, gait pattern recognition, and biofeedback approaches for lower extremity robotic prosthetics and orthotics. Finally, a recent systematic review on wearable ankle rehabilitation robots for post-stroke rehabilitation focused on actuation technologies, gait event detection, control strategies, and the clinical effects of the robotic intervention [59].

In this systematic review, we aim at complementing previous literature surveys by providing an updated structured framework of current control strategies, analyzing the methodology of clinical validations used in the robotic interventions, and reporting the potential relation between the employed control strategies and clinical outcomes. In this literature survey we seek to answer the following three research questions: (1) Which control strategies have been used on powered lower limb exoskeletons for people with brain injuries?, (2) What are the experimental protocols and outcome 
metrics used in the clinical validation of robotic interventions?, and (3) What is the current clinical evidence on the effectiveness of the different control strategies?

\section{Methods}

Search Strategy

To answer the first research question -i.e., which control strategies have been used on powered lower limb exoskeletons for people with brain injuries?- we conducted a literature search on the 17th of September 2020, including English-language studies published from January 2000 to September 2020 in four databases: Web of Science, Scopus, PubMed, and IEEE Xplore. The search included the following keywords: ("brain injury" OR "cerebral" OR "palsy" OR "stroke" OR "hemipare*" OR "hemiplegi*" OR "CVA" OR "cerebrovascular accident" OR "cerebral infarct" OR "cerebral hemorrhage" OR "ABI" OR "acquired brain injury" OR "motor learning" OR "neuroplasticity" OR "neural plasticity" OR "neuroplastic") AND (("lower" AND ("limb*" OR "extremit*")) OR "walk*" OR "ambulat*" OR "gait") AND ("power*" OR "active" OR "robot*" OR "wearable") AND ( "assistive" OR "exo*" OR "exosuit" OR "exo-suit" OR "brace*" OR "ortho*") AND "control*".

The search query led to 1648 studies (991 after removing duplicates). After a title and abstract screening, the number of studies was reduced to 255 . Then, a full-text screening process was carried out with the following criteria: studies should (1) involve active orthoses/exoskeletons for lower-limb training, (2) provide technical details about the control strategy used, (3) validate the device on people with a brain injury, and (4) report biomechanical or clinical outcome metrics that allow for a comparison among different control strategies. The last condition was associated with the analysis of the clinical methodology followed in robotic interventions. After the full-text screening, a total of 159 publications were included in this review (see Figure 1), with a total of 43 different lower limb exoskeletons. The resulting studies will be used to answer the first two research questions outlined in this review. See Additional file 1 for a detailed list of the studies included.

\section{Clinical Comparison}

To answer the third research question -i.e., what is the current clinical evidence on the effectiveness of the different control strategies?- we conducted a stricter screening of the 159 publications focusing on the studies that performed an assessment before and after the robotic intervention; the studies that focused only on assessments during the robotic intervention while wearing the robotic device, or only immediately after a single training session were not included. This screening resulted in 73 publications (see Figure 1).

To perform an unbiased clinical comparison between different exoskeleton controllers, we subdivided the participants with stroke and CP into different subgroups, based on their impairment level and/or acuity before the robotic intervention. For the stroke group, we used three levels of acuity: acute $(\leq 2$ weeks from stroke onset), subacute ( $\leq 6$ months from stroke onset), and chronic ( $>6$ months from stroke onset). In the case of $\mathrm{CP}$, we followed the four levels of the Gross Motor Function Classification System (GMFCS) [63].

Applying a final screening process, we only compared controllers tested with participants that shared similar levels of impairment before the robotic treatment, i.e., similar scores in Functional Ambulation Category (FAC) and in the metrics mentioned in subsection Outcomes of interest for the clinical comparison. This resulted in the exclusion of six studies on people with acute [64], subacute [64-69] and chronic [69] stroke.

This final screening process led to 73 studies of which 57 studies included stroke survivors $(78.08 \%$ of the studies) and 16 children/adults with CP (21.91\% of the studies). From the 57 studies that analyzed the benefits of robotic exoskeleton lower-limb training on stroke survivors, five studies included participants with acute stroke, 13 studies with subacute stroke, and 42 studies with chronic stroke. From the 16 studies with children/adults with CP, five studies included participants with GMFCS I, 15 studies with GMFCS II, 12 studies with GMFCS III, and four studies with GMFCS IV. Note that the total number of studies is not equal to the sum of the studies divided per level of impairment and/or acuity as, in some studies, participants were pooled together independently of their acuity and GMFCS levels. See Additional file 2 for a detailed list of the studies included in the clinical analysis.

To further analyze, compare, and discuss the effectiveness of different control strategies, we also took into consideration: (1) the grade of evidence based on the type of intervention -e.g., (randomized) clinical trials or observational studies-, (2) the training duration of the robotic treatment -e.g., number of repetitions, number of sessions, and frequency of the training-, and (3) the number of participants who trained with each type of control.

Following the guidelines presented in [70,71], we considered that a study had a high level of evidence (level I study) when it was a Randomized Clinical Trial (RCT). When the study was a Clinical Trial (CT), we considered that its level of evidence was moderate (level II study). And finally, the level of evidence of 


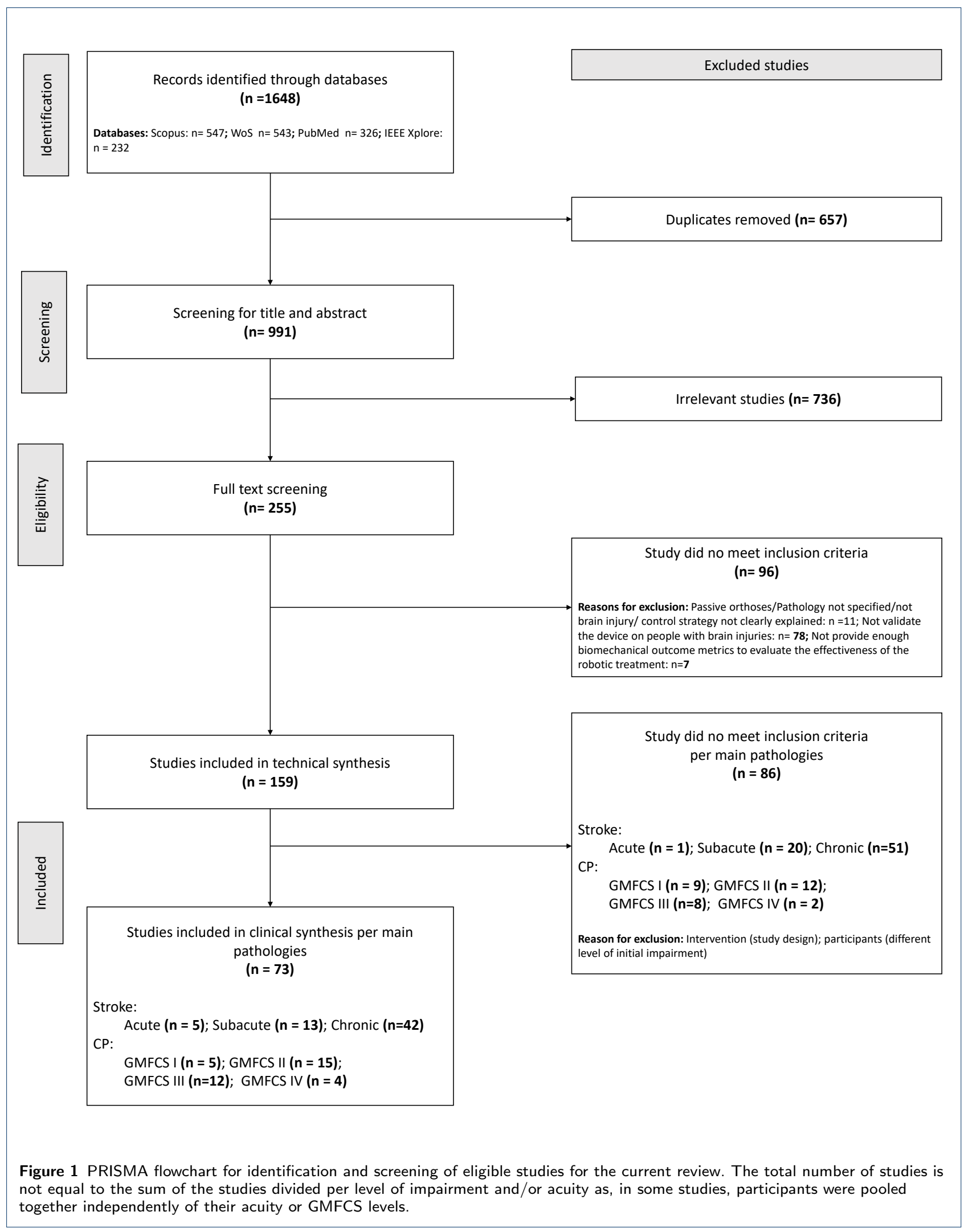


observational studies was considered as low (level III study). The grade of evidence of the clinical effects of the robotic treatment was considered as strong when there was a preponderance of level I and/or level II studies that supported the result -this must include at least one level I study. The grade of evidence was considered as moderate when there was a preponderance of level II and/or level III studies that supported the result -this must include at least one level II study. Finally, the evidence was graded as weak when only level III studies supported the result.

\section{Outcomes of interest for the clinical comparison}

The selected outcome measures of interest are based on those recommended by surveys and studies that evaluated stroke and CP rehabilitation [72-78]. Based on the literature, we have selected the following metrics to evaluate the effectiveness of different control methods on stroke survivors: Berg Balance Scale (BBS), 10 Meter Walk Test (10MWT), 6 Minute Walk Test (6MWT), Timed-Up and Go (TUG), Fugl-Meyer Assessment (FMA), and Functional Independence Measure (FIM) -this last one only for acute stroke. To evaluate the effectiveness of the control strategies on lowerlimb rehabilitation of participants with $\mathrm{CP}$, we selected the following scales: Gross Motor Function Measure (GMFM)-66/88 dimensions D and E, 10MWT, and $6 \mathrm{MWT}$.

\section{Control Strategies Taxonomy}

To analyze the state of the art of control strategies for lower limb exoskeletons in rehabilitation, we propose a hierarchical classification of control methods based on an adapted version of the categorization presented in [55]. The hierarchy establishes three different levels: High-level control, Mid-level control, and Low-level control (see Figure 2).

High-level controllers are defined as control strategies that identify the human's volitional intent and select the appropriate exoskeleton response behaviour. The exoskeleton Mid-level control reacts to the current state of the user and defines the reference position or force that the robot should follow based on the control aim and the state estimated by the humanrobot synchronization algorithm (both embedded in the High-level control) and the sensors measurements. The Low-level control tries to achieve the desired state determined by the Mid-level controller by applying feedforward or feedback control. In this systematic review, we have focused on High- and Mid-level controllers since they are highly related to exoskeleton use, while Low-level controllers are directly linked to the hardware and can be applied in other types of robots [41].
High-level control

A High-level control system provides a command that modifies the state of the actuation system in order to provide assistance or resistance to the user, depending on the control aim [79-81] (see Figure 3.A). The control aim varies the purpose of the exoskeleton based on the desired treatment approach. Assistive High-level controllers facilitate functional training by supporting the users' movements to complete the task -e.g., sit-to-stand [82], achieve stability during the loading response of the gait [83], or plantarflexion assistance in late stance [84]. It is thought that passively guiding movements may improve gait performance [85-87], especially in those suffering from severe impairment $[55,88]$. Additionally, passively mobilizing the affected limbs allows for stretching the muscles and might reduce spasticity [89], provides somatosensory stimulation that facilitates restoring normative patterns of motor output [87], and importantly, provides an environment for safe, high intensity, and motivating locomotion training. Yet, active participation is crucial for neuroplasticity $[90,91]$. For this reason, control strategies for people with brain injuries need to guarantee the patient's empowerment by providing progressive and tailored assistance or resistance.

On the contrary, Challenge-based High-level controllers aim at, e.g., strengthening the muscles by opposing to task completion - e.g., resistive methods [92], enhancing error detection -e.g., error augmentation methods [93]-, and increasing movement variability e.g., perturbation methods [94]. These challenge-based control strategies might lead to improvements in physical performance, movement control, walking speed, and functional independence, especially in people in the late stages of the rehabilitation or with mild impairment [95-98].

Evidence seems to indicate that the aim of the control strategy of an exoskeleton for people with brain injuries should be to stimulate physical/cognitive engagement and motor learning rather than enforce repetitive movements with low variability [21]. In particular, in people with moderate/mild brain injuries, excessive assistance may have a negative influence on motor learning, as the dynamics of the task to be learned is different from the trained task [99]. To promote users' active participation, the device should engage the users wearing the exoskeleton to, e.g., actively initiate each step or control their balance. Thus, generic controllers [100] that do not adapt their assistance/resistance based on users' needs might not be the most effective ones for gait rehabilitation of people with brain injuries who preserve partial or full volitional control [91, 101, 102]. Robotic training using adaptive controllers that modulate the assistance 


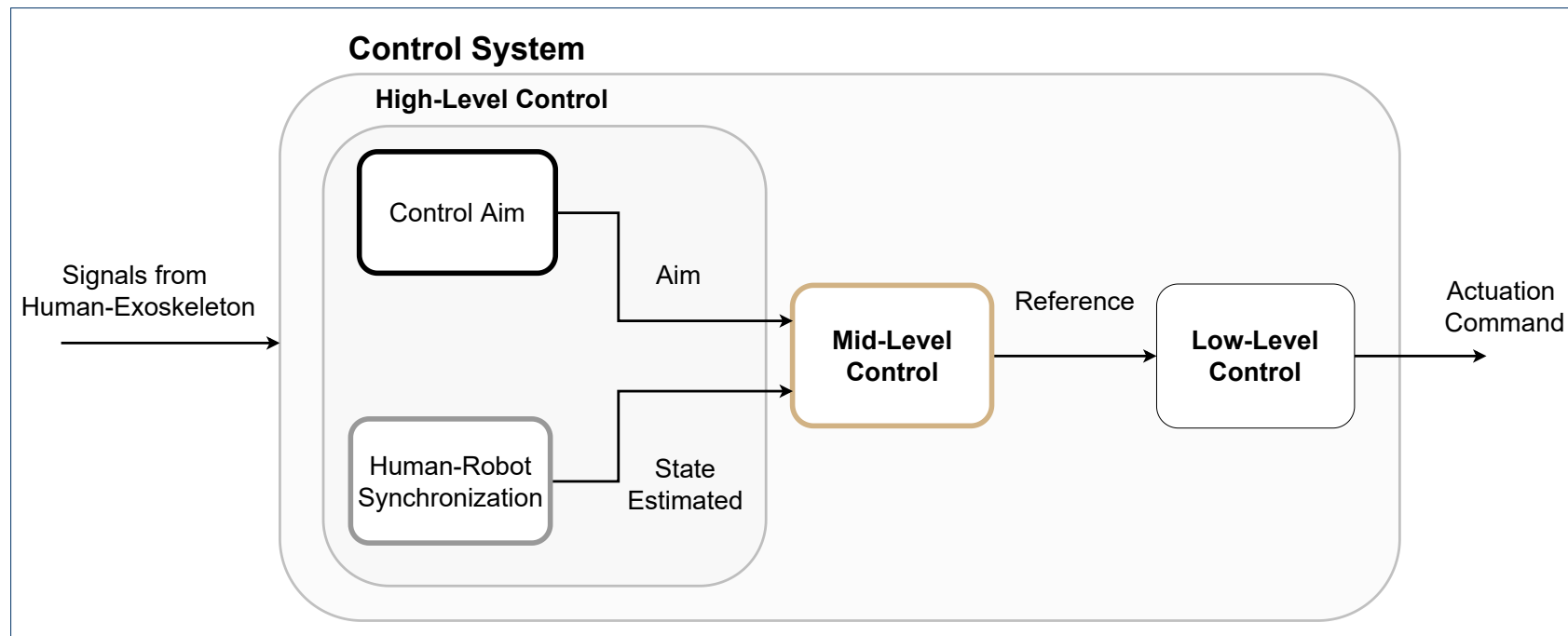

Figure 2 General Control System Diagram. The signals from the Human-Exoskeleton -e.g., human-robot interaction forces, limbs' kinematics, and/or recorded human muscle or brain activity- are processed sequentially by three different blocks -each corresponding to High-, Mid- and Lower-level control-, to generate the actuation command. High-Level Control: The Control Aim defines the role of the exoskeleton in the overall performance of the human-exoskeleton system, i.e., enhance or hinder task completion. The human-robot synchronization block generates an estimation of the actual state and is used by the Mid-level control strategy, together with the control aim, to provide reference values -e.g., desired position or force- to the Low-level controller. The Low-level controller then transforms that reference into actual assistive/resistive force/motion and sends the actuation command to the exoskeleton hardware.

based on users' performance might be more effective to stimulate motor learning than those that enforce generic "normative" movements independently of the users' capabilities [103].

To maximize the users' physical and cognitive engagement/effort and prevent "slaking" [104], adaptive strategies provide tailored assistance or resistance based on real-time biomechanical measurements during locomotion (e.g., joint kinematics [105] and ground reaction forces [92]). The controller adaptation to the user's specific needs might be done by modifying the parameters of a reference trajectory [106-108] or the dynamics of a virtual compliant model [109-111].

Synchronization to the user's motion is a key factor to effectively benefit from the exoskeleton therapy, e.g., reducing adaptation time and metabolic rate [100]. Most of the Mid-level control strategies need an estimation of the current action performed by the user to properly assist or resist her/his motion, i.e., to synchronize the human and the robot. The human-robot synchronization sub-level within the High-level control estimates the state of the user by using deterministic or stochastic methods based on recorded kinematic, kinetic, and/or bioelectric data -e.g., joint kinematics [112], ground reaction forces [113], human-robot interaction forces [106], muscular activity [114], and brain activity [115]- (see Figure 3.B).

Threshold-based algorithms differentiate between states -e.g., gait phases [116], falling [117], and startstop walking [118]-following a state-machine structure that allows the transition between states depending on logical rules.

Stochastic algorithms, on the other hand, infer the state throughout statistical models, e.g., using Linear Discriminant Analysis (LDA) [115], Hidden Markov Models [119], Principal Component Analysis [120], KNearest Neighbours [121], or Neural Networks [122]. This family of human-robot synchronization methods is particularly useful to plan the gait pattern of the exoskeleton based on vision-based environment classification due to the high performance of stochastic algorithms to classify environments using images [123].

Bio-inspired models are emerging as an alternative to threshold-based and stochastic algorithms. For example, adaptive oscillators are non-linear models that synchronize with a teaching signal -e.g., the thigh angle in the sagittal plane [124]- in phase, frequency and amplitude, mimicking bio-inspired behaviours [125]. The estimated output from the adaptive oscillator e.g., phase of the input signal- is used to estimate the phase of the gait or to generate reference joint trajectories to assist or resist the human motion $[124,126,127]$. The main disadvantage of adaptive oscillators, however, is that they require a precise parameter tuning to quickly synchronize with the human periodic motion [128].

Nevertheless, all human-robot synchronization methods require a parameter tuning to properly adapt to each specific user's gait as they are not generalizable 
A.

I.

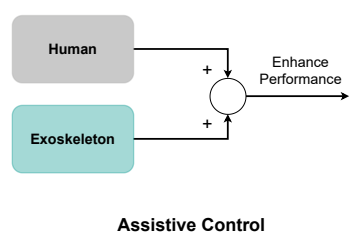

II.

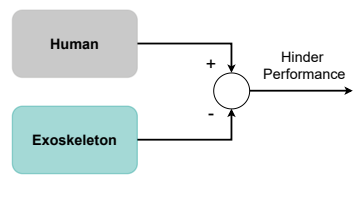

Challenge-Based Control
III.

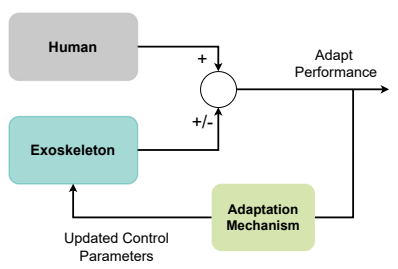

Adaptive Control

Control Aim

B.

I.

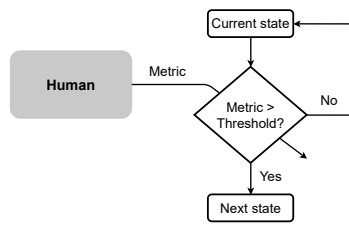

Threshold-based Algorithm
II.

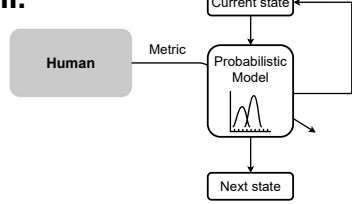

III.

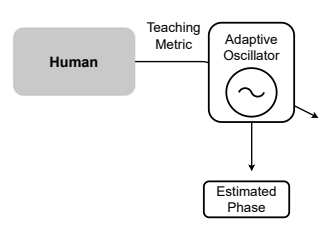

Stochastic Algorithm

Adaptive Oscillator

Human-Robot Synchronization

C.

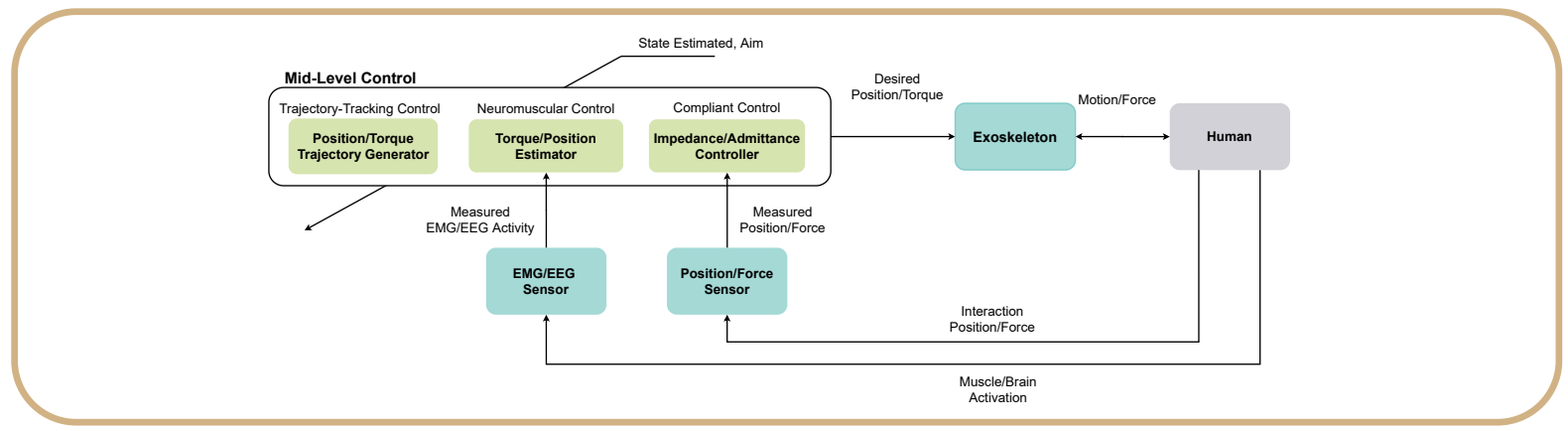

Mid-Level Control

Figure 3 Taxonomy of High- and Mid-Level controllers. (A) Control Aim: (A.I) In assistive control, the exoskeleton provides support to enhance the movement performance during training. (A.II) Conversely, in challenge-based control mode, the exoskeleton provides actions that hinder the human performance. (A.III) Adaptive control adjusts the system parameters based on the human-robot performance to provide adjusted assistance or resistance. (B) Human-Robot Synchronization: (B.I) Threshold-based algorithms ensure the transition between states whether the detection metric fulfils a pre-defined threshold. (B.II) In stochastic algorithms, the transition between states for the same set of initial conditions and algorithm parameters might be different due to the inherent randomness of the models used. (B.III) Adaptive oscillators use the periodic motion of the user to extract its phase either to generate a control signal or to determine the actual state of the user, e.g., the phase of the gait. (C) We categorize the Mid-level control strategies used in lower-limb exoskeletons for gait rehabilitation into three families: Trajectory-tracking control generates reference assistive or resistive torque/position profiles based on parameterized or pre-recorded position/torque trajectories; neuromuscular control uses recorded biosignals (e.g., brain/muscle signals) to generate the control signal for the Low-level control; and compliant controllers regulate the impedance or admittance of the exoskeleton by modifying the dynamic relation between movement and force or force and velocity respectively.

enough to avoid patient-to-patient variability [129]. This process is laborious, as therapists must manually tune the parameters offline relying only on feedback from the patients and subjective visual assess- ments [130,131]. Automatic adaptation [132] based on 388 the users' intention and/or gait parameters, such as 389

gait speed [133-135], might facilitate the usability of 390 these methods. 


\section{Mid-level control}

Mid-level control employs sensor measurements, the control aim, and the state inferred by the humanrobot synchronization to generate reference control commands used by the Low-level control to apply the actuation command (see Figure 2). Three different families of Mid-level control strategies can be distinguished depending on the control inputs/outputs and controllers employed (see Figure 3.C).

Trajectory-tracking control generates predefined position or force trajectories as reference commands to provide assistance/resistance. These trajectories are usually determined based on pre-recordings of unimpaired individuals (e.g., hip and knee flexionextension, and ankle plantarflexion-dorsiflexion torques [136]), information from the non-paretic limb (e.g., hip and knee flexion-extension angles [112,137]), or pre-recorded trajectories during therapist-guided assistance (e.g., foot trajectory [138] or knee flexionextension [139]).

Neuromuscular control strategies use biosignal recordings as control signals to decode the actions of the user and send reference values to the Low-level control [140]. Common approaches, like myoelectric [107, 141, 142] and Brain-Computer Interface (BCI) [143, 144] control, use muscular -electromyography (EMG) - and brain - electroencephalography (EEG)signals, respectively, to handle the control objective.

Lastly, compliant controllers [145, 146] regulate the impedance $[119,147]$ or admittance $[148,149]$ levels of the exoskeleton by modifying the dynamic relation between movement and force or force and velocity, respectively, using virtual dynamics of springs, dampers, or masses. The combination of trajectory-tracking control [105] or neuromuscular control [150] with compliant control usually provides a more flexible behaviour to the exoskeleton during rehabilitation -e.g., by allowing more movement variability around the desired trajectory-, compared to conventional rigid Low-level controllers such as proportional-derivative (PD) controllers [151, 152].

\section{Review}

Implementation of Control Strategies

In this section we provide an overview of the High and Mid-Level control strategies implemented in the studies included in this review from a technological point of view, without focusing on clinical aspects (see Figure 4.A). Exoskeletons used with people with stroke and cerebral palsy are highlighted as these two were the most predominant pathologies in the reviewed studies (see Figures 4.B-C).
High-Level Control: Control Aim

The majority of the exoskeletons validated on stroke survivors $(78.9 \%)$ and children/adults with cerebral palsy $(50.0 \%)$ implemented assistive strategies that adapted their behaviour depending on the user's intention and/or capabilities. The controller adaptation to the user's specific needs was mainly done by modifying the reference trajectory $(67.4 \%$ for all the pathologies, $71.0 \%$ for stroke and $50.0 \%$ for CP) or the dynamics of a compliant controller $(6.9 \%$ for all the pathologies, $7.9 \%$ for stroke and $0.0 \%$ for $\mathrm{CP}$ ). A majority of the exoskeletons adapted online either the reference trajectories [106-108] or parameters of the compliant models [109-111] based on real-time measurements of the patient's biomechanics, e.g., the ankle angle tracking error [153], gait speed [107], or ground reaction forces [154]. There were also a few examples of devices that tuned the timing and magnitude of the assistance offline based on the patient's motor function, previously assessed by the therapists $[129,131,155,156]$.

Only $10.5 \%$ of the exoskeletons for stroke rehabilitation and $20.0 \%$ for cerebral palsy validated challenged-based control strategies, e.g., using resistive forces [157-159], perturbing forces [94], or haptic error augmentation [160]. All the challenge-based control strategies $(11.6 \%$ of the exoskeletons for all pathologies) implemented in these studies were adaptive as they modified their behaviour based on the user's gait performance -e.g., based on the tracking error $[159,160]$, or ground reaction forces [157]- or applied the control action on participant-dependent instants of the gait cycle $[94,158]$.

Notably, none of the reviewed studies adapted the assistance/resistance based on direct gait biomechanical descriptors of the brain-injured population. This might be due to the small number of reviewed studies that analyzed the effect of the control parameters on the participants' gait kinematics and kinetics $[108,153,155,161-163]$. Besides, the majority of these few studies only focused on analyzing the effect of the timing and magnitude of the assistive torque or position trajectories on ankle power [155], walking speed, step length, joint kinematics [108, 162, 163], metabolic cost, or muscular activity [161]. Only one study explored the effect of varying the parameters of an impedance model on the ankle position on the sagittal plane [153]. Yet, biomechanical metrics -e.g., step length [164], hip hiking [165], and trailing-limb angle during the stance phase [166]- might more directly reflect the user's rehabilitation progress.

High-Level Control: Human-Robot Synchronization Threshold-based approaches were the most implemented human-robot synchronization algorithms on 
A.
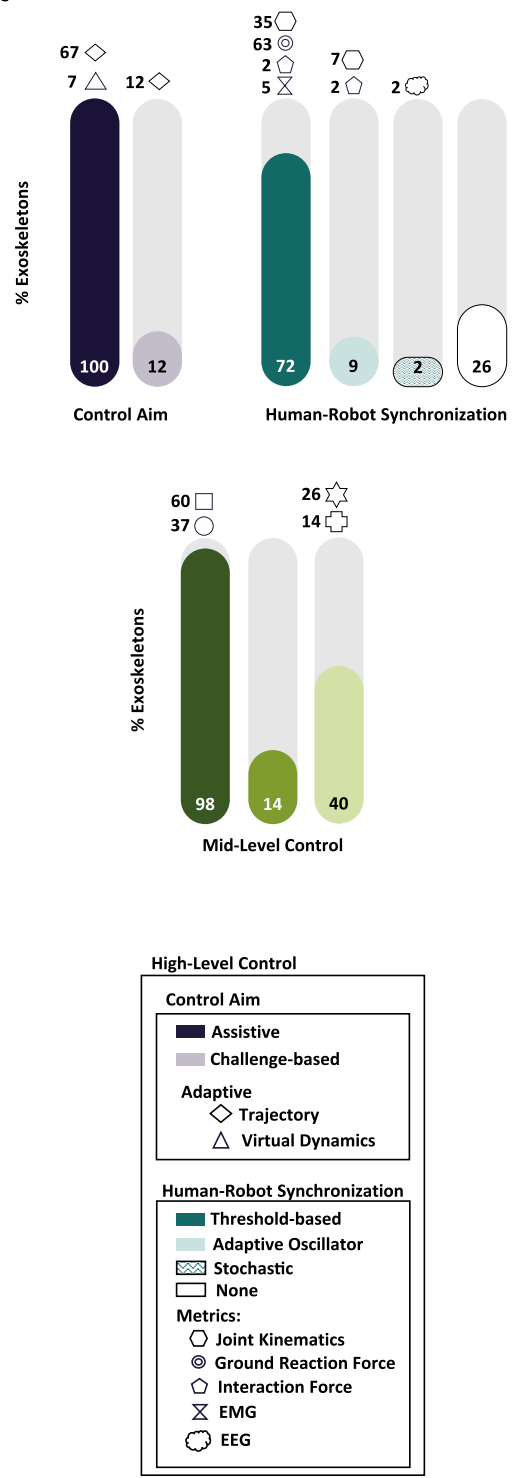

\begin{tabular}{|} 
Mid-Level Control \\
\hline Trajectory-Tracking \\
$\square$ Position \\
O Force \\
Myoelectric \\
Compliant \\
5 Impedance \\
$\square$ Admittance \\
\hline
\end{tabular}

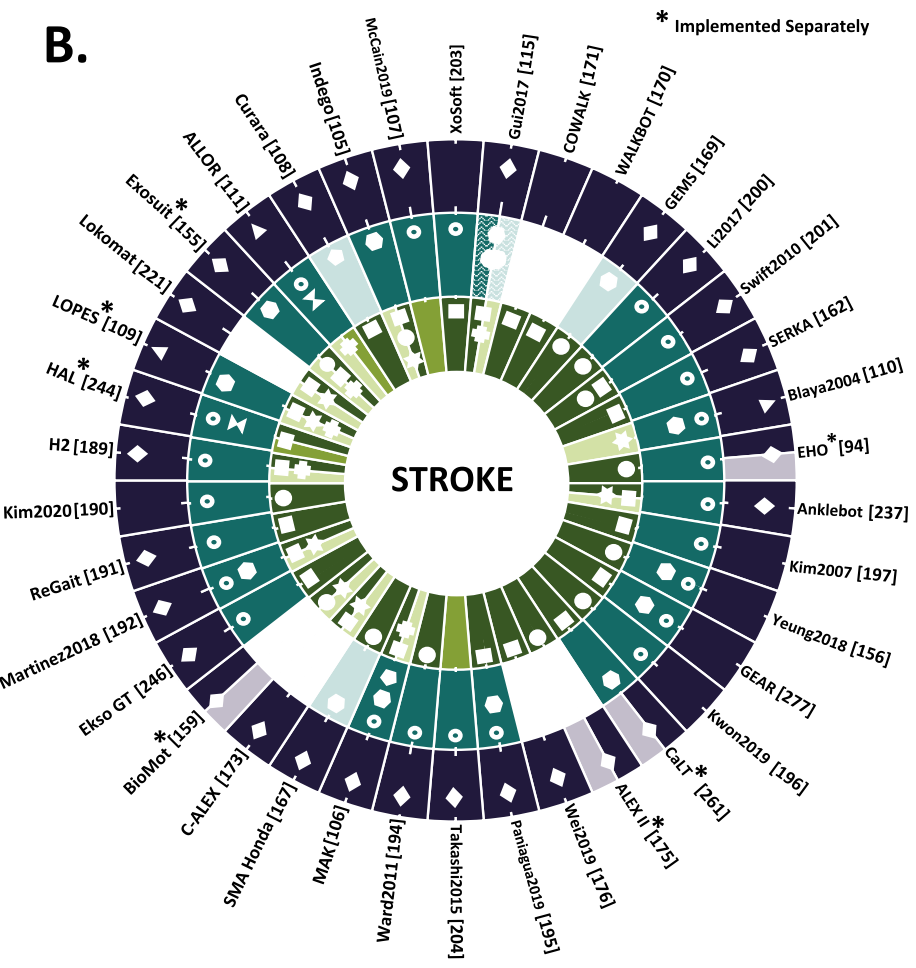

C.

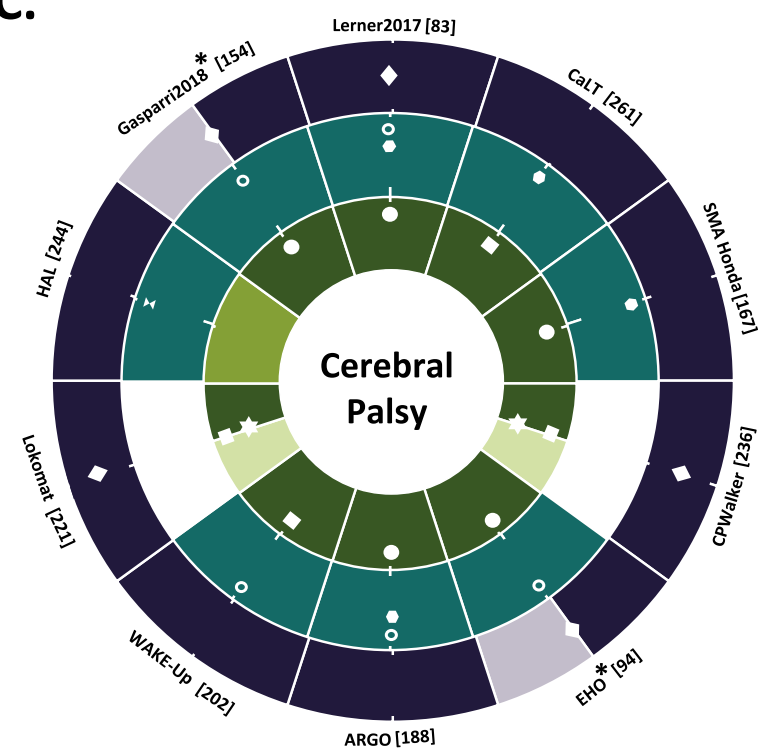

Figure 4 Overview of exoskeletons based on their High- and Mid-level control strategies. Each color represents the different families inside the High- and Mid-level controllers and the symbols point out the categories inside these families. (A) Percentage of exoskeletons that implemented the different families and categories of High- and Mid-level controllers for all the pathologies included in this review. Note that the the same exoskeleton could incorporate different controllers, and therefore, the summation of percentages can be higher than 100\%. (B-C) Circular plots illustrate the High- and Mid-level control strategies of exoskeletons tested on people with stroke (B) and cerebral palsy (C). Each circular sector represents a different exoskeleton and every ring represents different levels of the control hierarchy. The outer ring is the control aim, the middle ring is the human-robot synchronization, and the inner ring is the Mid-level control. If a symbol lies in the middle of a subdivision within a sector, it implies that the characteristic related to that symbol applies to both subdivisions. 
lower-limb exoskeletons for people with brain injuries in general (72.1\% of exoskeletons), and stroke survivors (73.6 \% of the exoskeletons) and cerebral palsy participants (80.0\% of the exoskeletons) in particular.

Adaptive oscillators were tested with people with stroke in four different exoskeletons (10.5\% of the exoskeletons) using sagittal lower-limb segment angles, joint angles, or robot-human interaction forces as synchronization signals $[108,167-169]$.

A few number of devices $(25.6 \%$ ) did not implement any type of event detection algorithm for humanrobot synchronization, probably because they did not strictly need it [170-176]. Most of them were grounded exoskeletons that either enforced joint angle reference trajectories during gait -based on the unimpaired joint movement- using non-adaptive assistive control strategies $[170,171]$, or employed an adaptive assistive controller around the desired trajectory [172-176].

Only one exoskeleton in this review implemented stochastic methods to distinguish between different locomotion modes, i.e., stop, normal walk, acceleration, and deceleration [115]. They used linear discriminant analysis (LDA) with EEG signals to differentiate between the frequencies of the brain activity associated to each mode.

We consider that two main reasons may have led to the lack of implementation of stochastic methods: (1) having a stochastic model that is flexible and able to capture the variance of the population (i.e., does not underfit) requires training data that captures the heterogeneity of people with brain injuries, which might be difficult to obtain [177]; and (2) the difficulty of getting robust stochastic models hinders their application in commercial exoskeletons, as regulatory bodies impose strict safety standards to validate such devices for clinical use [178].

Exoskeletons and prosthesis share similar challenges in terms of human-robot synchronization, but in the case of prosthetic devices, the tendency to apply stochastic methods is higher than using thresholdbased approaches $[179,180]$. This might be explained by the homogeneity in the gait of amputees compared to the heterogeneity observed in people with brain injuries [181-183]. Nevertheless, as in the case of lowlimb exoskeletons, there is a lack of use of stochastic methods in commercially available prostheses [184].

We have not found any exoskeleton in the framework of this review that implements algorithms that automatically adapt the threshold values or model parameters related to gait event identification algorithms. Gait state detection methods with the ability to adapt to diverse walking conditions, e.g., different cadences [185], are still pending to be implemented and validated on exoskeletons for people with brain injuries.
The most common metric used to detect gait events was the vertical ground reaction force $(62.8 \%$ for all the pathologies, $60.5 \%$ for stroke and $50.0 \%$ for CP), probably due to its simplicity in the theoretical and practical implementation [186]. Ground reaction forces are directly related with the physics of foot-ground interaction. Normal or vertical force component is the one that allows to identify the phases of the foot contact and lift. Force-sensing resistors, placed at particular foot locations -e.g., heel, toe, and first and/or fifth metatarsals-, were generally used to measure this metric [94,106,110,111,161,163,176,187-203]. Alternatively, instrumented treadmills were employed to measure anterior-posterior ground reaction forces to determine the timing of the ankle plantarflexion assistance $[107,204]$. However, the suitability of this metric to treat people with brain injuries is questionable due to their irregular center of pressure trajectory along a walking cycle. The lack of uniformity might come from equinovarus deformity [205], excessive hip external rotation [16, 206], or reduced propioception [207, 208]. Thus, it might be challenging to develop robust gait event detection algorithms that use ground reaction forces for this specific population.

Human-robot interaction forces have only been implemented on two exoskeletons $(4.6 \%)$. In the first exoskeleton, the human-robot interaction forces were employed to feed a threshold-based algorithm to detect the swing phase [106], while in the second exoskeleton they were used as the teaching signal of a pool of adaptive oscillators [108]. Only a few devices used human-robot interaction forces as control inputs $[106,115,155,189,209]$, which might explain why the use of this metric to detect actions or states is scarce in exoskeletons for people with brain injuries. The mechanical adaptation of the exoskeleton required to directly measure this metric might be behind the rare use of this metric.

Only a few reviewed studies incorporated biosignals as metrics in their human-robot synchronization algorithms $(4.6 \%$ of the exoskeletons for all the pathologies). For example, EEG was used by only one exoskeleton [115] to detect different locomotion modes, i.e., stop, normal walk, acceleration, and deceleration. Problems related to EEG analysis, such as feature extraction and artifact removal [58, 210, 211], might make the implementation of reliable control strategies a challenge. Furthermore, EEG-based synchronization might require high levels of attention from the patient, which might result in fatigue [212], and thus, might limit the training duration. Nevertheless, brain activity might be especially useful for individuals who suffer from a severe neurological condition, such as paraplegia $[213,214]$. 
In people who preserve their voluntary muscle control over the affected limbs, muscular activity might be a more suitable metric compared to brain activity. Yet, only two devices $[111,114]$ validated muscular activity as an event detection metric in people with brain injuries. These devices employed muscular activity (EMG) from the trunk, hip, and knee flexor/extensor muscles to trigger the control action. There are several limitations associated with the use of muscular activity to detect gait events. First, surface electromyography (sEMG) signals suffer from non-robustness due to patient-to-patient variability and sensor-placement dependency $[38,59]$. Moreover, muscular activity might not be reliable in individuals who have abnormal muscle activation patterns, such as stroke and $\mathrm{CP}$ survivors $[58,215]$.

We consider that joint or body segment kinematic metrics (used in $41.8 \%$ of the exoskeletons for all the pathologies, $36.8 \%$ for stroke, and $40.0 \%$ for CP) might be more reliable metrics in human-robot synchronization algorithms when detecting events with brain-injured people [216], as higher homogeneity in kinematic metrics can be found among people with hemiplegic gait $[217,218]$. In particular, the shank absolute angle and angular velocity in the sagittal plane have been shown to be especially robust metrics to detect gait events in people with hemiplegic gait [219].

\section{Mid-Level Control}

Trajectory-tracking control is the most used Midlevel control strategy in lower limb exoskeletons for rehabilitation $(97.7 \%)$. The most common approach is to enforce predefined reference position or torque trajectories defined based on data of unimpaired joints $[69,191,220]$. Trajectory-tracking control was combined with compliant control $(28.9 \%$ of the exoskeletons for stroke and $20.0 \%$ of the exoskeletons for $\mathrm{CP}$ ) in assistive controllers based on potential $[102,106,175,189,221]$ or velocity fields [105]. In these examples, the assistive action of the exoskeleton varied based on the joint kinematic errors.

Only four devices (13.9\% of the exoskeletons) that used myoelectric control were validated on people with brain injuries [66, 107, 111, 204]. Myoelectric control is one of the least often employed mid-level control strategies in post-stroke (10.5\% of the exoskeletons) and cerebral palsy (10.0\% of the exoskeletons) rehabilitation, according to the results of this review. The aforementioned issues with muscle activity recording and analysis (see High-Level Control: Human-Robot Synchronization for a detailed discussion) might be behind the low adoption of this mid-level control technique. Nonetheless, myoelectric control has a high applicability for people who preserve volitional control of the muscles, such as users of robotic prosthetic devices [222].

None of the reviewed studies incorporated BCI control with people with brain injuries. Problems related to the extraction of relevant information from, e.g., EEG recordings (see High-Level Control: HumanRobot Synchronization for a detailed discussion) might also explain the lack of usage of this Mid-level control technique in exoskeletons for people with brain injuries. EMG is a viable alternative or adjunct to EEG for detecting movement intention or generating control signals, but the practical benefits of using EMG over EEG, e.g., shorter set-up time, more compactness, and lower doning/offing times, might explain why myoelectric control has been more often used than BCI control [214]. Few studies, aside the ones included in this review, evaluated the feasibility of using EEG signals for BCI control of exoskeletons for people with brain injuries without implementing the BCI controllers on the devices $[223,224]$.

\section{Clinical Validation}

This section provides an overview of the most important characteristics of the clinical validation of the robotic interventions, i.e., participants' demographics, protocol design, and outcome measures. The results summarized in this section only incorporate participants who tested the exoskeletons and not participants in the control group. See Additional file 1 to have a more detailed description about the studies included in the clinical validation.

\section{Participants' Demographics}

Stroke was the main pathology of the participants recruited for the studies included in this review ( $74 \%$ of the studies) (see Figure 5.A). The majority of the participants with stroke were in the chronic phase $(55.41$ $\%$ of participants with stroke), followed by subacute $(33.83 \%)$ and acute $(10.76 \%)$ phases. Cerebral Palsy was included in only $20 \%$ of the studies, while the representation of other brain injuries, like traumatic brain injury $(1.2 \%)$ or acquired brain injury $(1.88 \%)$, was scarce. It is especially remarkable that despite the high incidence of traumatic brain injury, only two studies focused on this specific population $[225,226]$.

\section{Experimental Protocol}

High variability was found in the number of partici- 702 pants $(14.87 \pm 13.53)$, number of sessions $(11.77 \pm 12.20), 703$ session frequency (times per week; $3.09 \pm 1.68$ ), and 704 session duration $(50.57 \pm 34.06 \mathrm{~min})$ (see Figure 5.C). $\quad 705$ Previous reviews that analyzed the protocol of robotic 706 treatment reported similar high variability $[40,46] . \quad{ }_{707}$ Some studies did not provide complete information 708 05 06 
about the experimental protocol, e.g., they did not mention the number $(15.09 \%)$, duration $(33.33 \%)$, or frequency $(31.44 \%)$ of the training sessions.

Free walking without the exoskeleton was the condition most often employed to compare the robotic treatment with (39.62 \%) (see Figure 5.B). There were also studies that compared the robotic treatment with conventional gait therapy $(22.01 \%)$, while other studies compared the robotic treatment with the effect of using the device unpowered $(10.69 \%)$ or in zero torque mode $(6.92 \%)$.

The average level of evidence of the studies included in this review was low. The majority of the studies were observational $(66.04 \%)$, while only $10.06 \%$ and $22.64 \%$ were CTs and RCTs, respectively. Only 12.58 $\%$ of the studies did a follow-up evaluation after the robotic intervention, on average four months after the last intervention.

\section{Outcomes of Interest}

Ambulation scales were the main metrics used to classify the initial functional level of participants for all the studies. The participants' baseline was determined using metrics that analyzed their level of impairment and motor function -GMFM (19.50\%), FMA (13.21\%), and Brunnstrom Stage (BS) (7.55\%)-, mobility -TUG (10.06 \%), FAC (29.56 \%), BBS (14.47 \%)-, spasticity -modified ashworth scale (MAS) (15.09\%)-, and functional capacity and activities of daily living -walking speed (56.6\%), 10MWT (20.75\%), 6MWT (16.98\%), FIM $(8.81 \%)$, and Barthel Index (BI) (11.32\%)- (see Figure 6.A).

A critical limitation we encountered when comparing robotic treatments was the low homogeneity across studies in the selected outcome measures after the treatment, as no metric was used in more than $50 \%$ of the studies (see Figure 6.B). Ambulation scales together with spatio-temporal parameters were similarly used to determine the effect of the robotic treatment (62.89\% of the studies). Within these families of metrics, gait speed was the most used metric in the reviewed studies (37.74\%), followed by cadence (25.16 $\%)$, and step length $(23.27 \%)$. Joint kinematics was also often used to quantify the effect of the robotic intervention $(44.65 \%)$. Hip $(22.64 \%)$, knee $(27.67 \%)$, and ankle $(18.87 \%)$ ranges of motion (RoM) in the sagittal plane were the most often selected kinematic metrics.

Finally, the number of studies that analyzed the muscular activity through sEMG was lower in comparison with the aforementioned families of metrics $(20.75 \%)$. The main analyzed muscles were the ankle dorsiflexor (tibialis anterior, $10.69 \%$ ) and plantaflexor (gastrocnemius, $8.81 \%$; and soleus, $6.92 \%$ ) muscles, and the knee extensor (rectus femoris, $9.43 \%$; and vastus lateralis, $5.03 \%$ ) and flexor (semitendinosus, $6.92 \%$ ) muscles. Less frequently employed metrics include those related to gait dynamics $(18.23 \%$, where the most used was ankle torque in $5.03 \%$ of the studies) -i.e., joint torques and ground reaction forces-, energy expenditure $(10.69 \%$, where the most used was oxygen consumption in $5.66 \%$ of the studies), and neural activity, i.e., brain activation and cortex excitability $(6.29 \%)$.

\section{Clinical Comparison of the Control Strategies}

This section quantifies the relation between the control strategies and the clinical metrics presented in the subsection Outcomes of interest for the clinical comparison to compare among strategies.

Based on the included studies, we could only extract moderate conclusions from the studies that involved post-stroke participants. The studies that involved patients with $\mathrm{CP}$ or traumatic brain injury, did not allow for a comparison of the control strategies implemented, due to the lack of studies with exoskeletons using different control strategies. For the case of $\mathrm{CP}$, in the studies that incorporated the main outcomes of interest, participants were pooled together, independently of their GMFCS level [227-230]. Only in a few studies that used the Lokomat [231-235] and CPWalker [236] the outcomes of interests selected in Outcomes of interest for the clinical comparison were evaluated and differentiated between different GMFCS levels. However, those studies implemented the same family of control strategies, namely adaptive assistive control strategies without human-robot synchronization algorithms that combined trajectory-tracking and compliant control, and thus, no comparison between controllers was possible.

Regarding the experimental protocol of the studies included in this section, we observed similar high variability in the number of participants and training duration than that found for the studies included in the previous section (see subsection Experimental Proto$\mathrm{col})$.

See Additional file 3 for a detailed table of the control strategies implemented in the reviewed studies and the results obtained in the main outcomes of interest for people with stroke.

\section{Acute Stroke}

From the originally listed outcome metrics of interest, FIM was the only metric that allowed an unbiased and direct comparison of the effectiveness of different control strategies in acute stroke rehabilitation [142,237-240]. The participants included in the considered studies (35.80 \pm 22.07 participants) presented an average initial FIM score of $2.5 \pm 1.29$ and 
A.

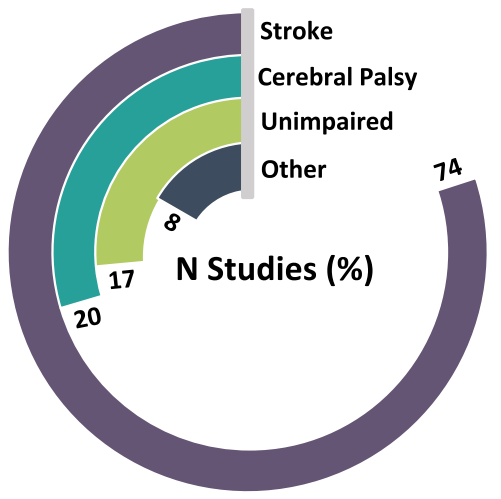

Pathologies

C.
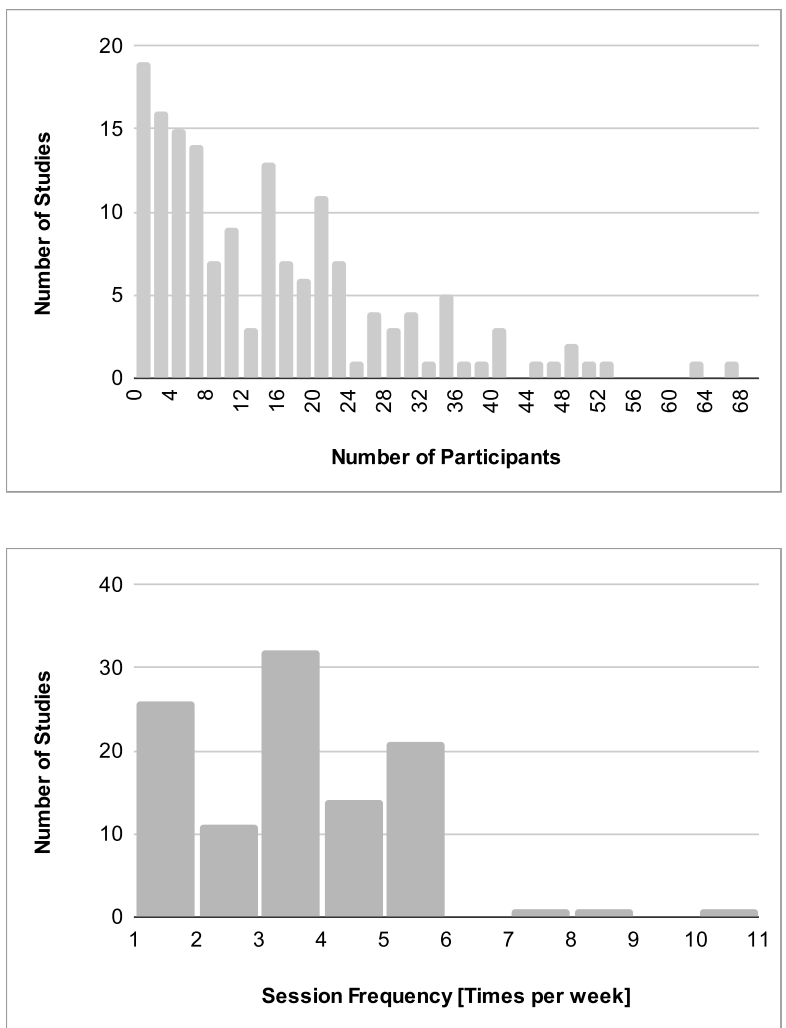

B.

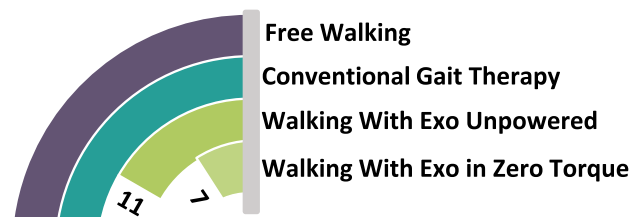

N Studies (\%)

22

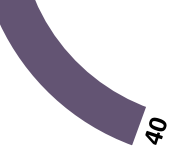

Main Control Conditions
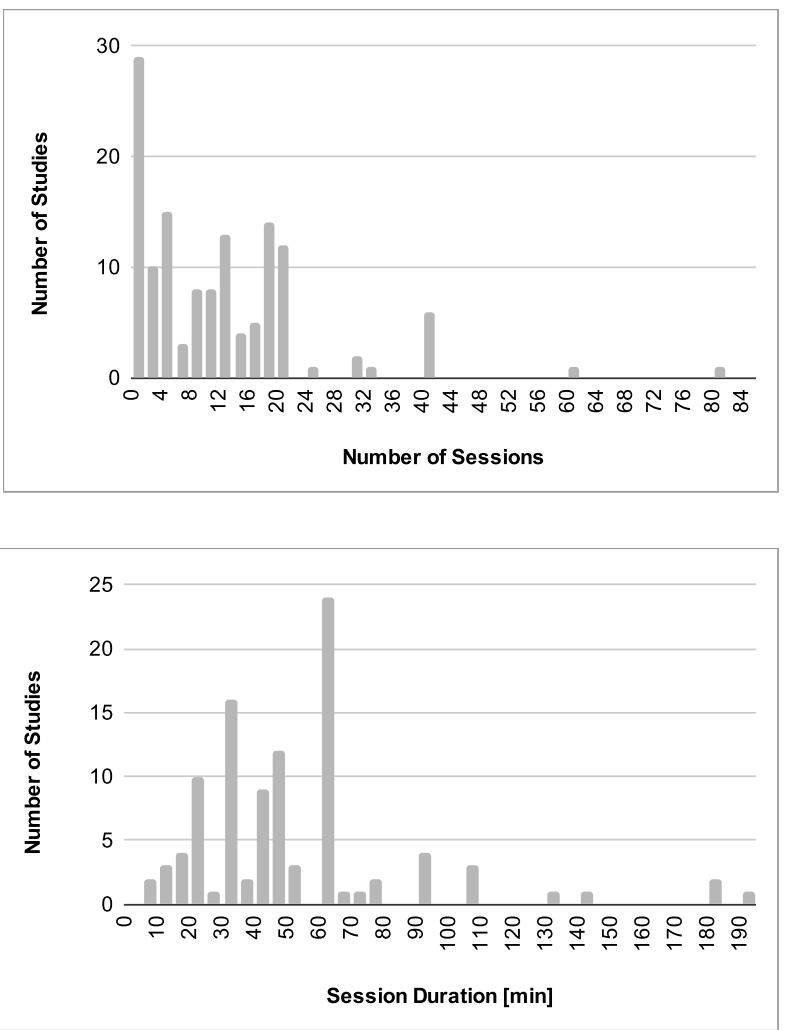

Figure 5 Overview of the participants' demographics and experimental protocol characteristics. (A) Percentage distribution of the pathologies of the participants included in the reviewed studies. (B) Percentage distribution of main control conditions in the studies. (C) Histograms of number of participants (top left), number of sessions (top right), sessions frequency (times per week; bottom left), and session duration (bottom right) across the selected studies.

an average training time of the robotic intervention of $360 \pm 244.95 \mathrm{~min}$.
Adaptive assistive control strategies that implemented a combination of trajectory-tracking and com- 


\section{A.}

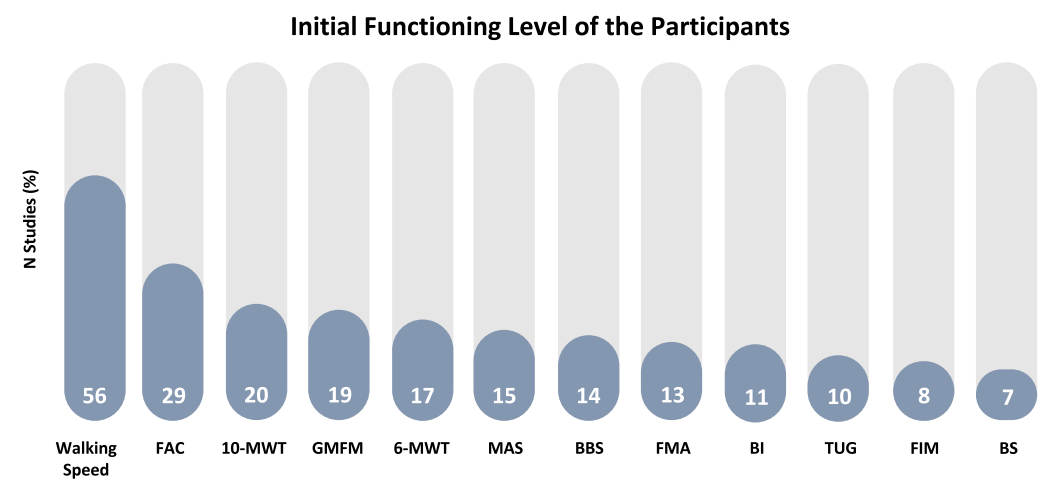

B.
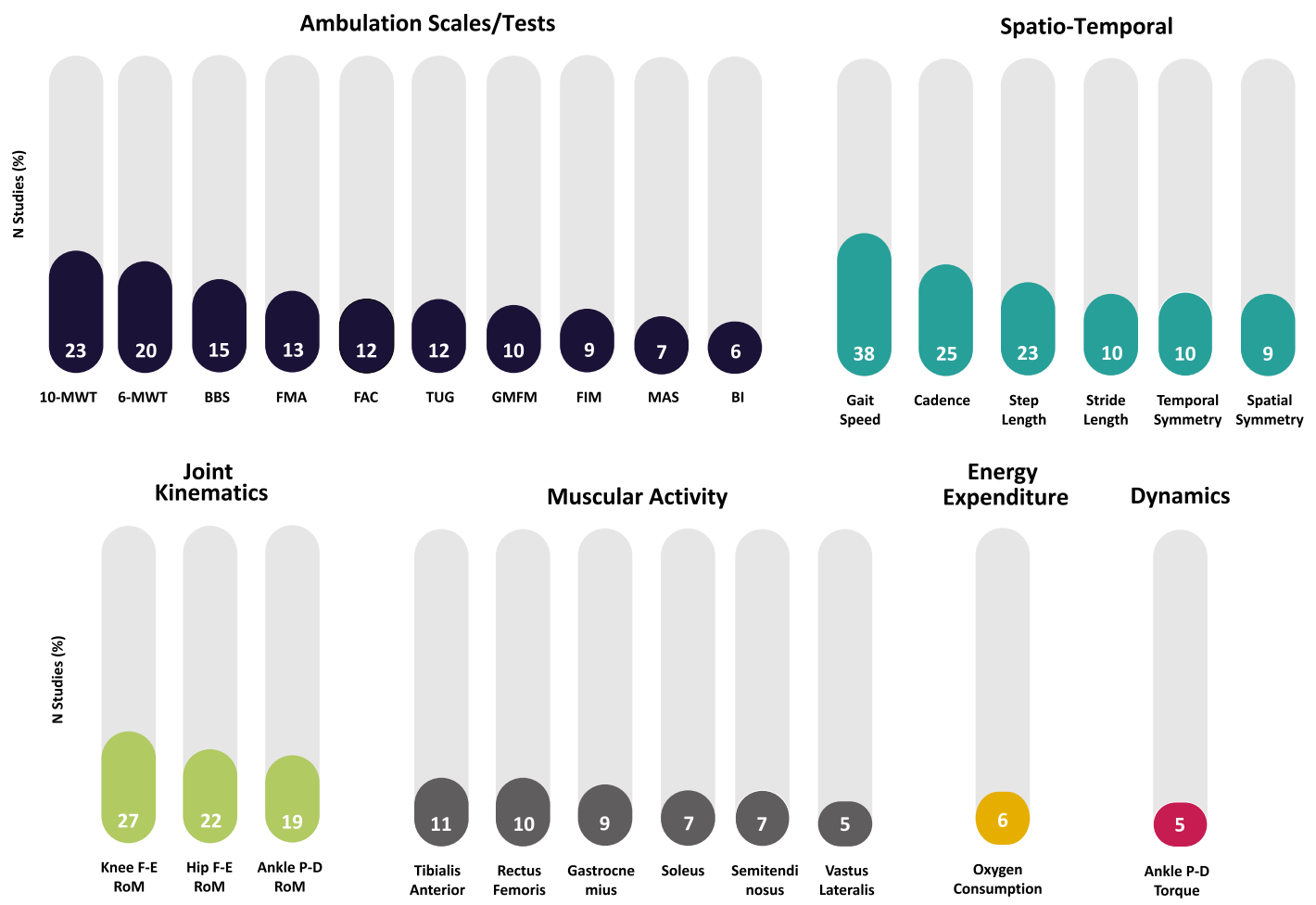

Figure 6 Overview of the main baseline and outcome metrics. (A) Percentage distribution of the metrics that were used in at least $5 \%$ of the studies to determine the initial functioning level of participants. (B) Percentage distribution of the outcome measures of the robotic interventions that were used in at least $5 \%$ of the studies grouped by categories, i.e., ambulation scales/tests, spatio-temporal measurements, joint kinematics, muscular activity (EMG), dynamics, and energy expenditure. Functional Ambulation Category (FAC), 10 Meter Walk Test (10MWT), 6 Minute Walk Test (6MWT), Gross Motor Function Measure (GMFM), Modified Ashworth Scale (MAS), Berg Balance Scale (BBS), Fugl-Meyer Assessment (FMA), Barthel Index (BI), Timed-Up and Go (TUG), Functional Independence Measure (FIM) and Brunnstrom Stage (BS).

pliant Mid-level control showed an improvement after training of $272.73 \%$ in FIM [237] with a strong grade of evidence. Conversely, non-adaptive assistive strategies that included a threshold-based algorithm based on EMG recordings as detection metric and control sig- nal showed an improvement after training of $58.33 \%{ }_{823}$ in FIM with moderate grade of evidence [142,238-240]. $\quad{ }_{824}$ However, the observed higher improvement in FIM in ${ }_{825}$ the adaptive assistive control strategies could also be ${ }_{826}$ explained by the longer training duration when using ${ }_{827}$ 
these adaptive techniques $(\approx 600 \mathrm{~min})$ compared to the duration of training with non-adaptive assistive strategies $(\approx 240 \mathrm{~min})$.

\section{Subacute Stroke}

The metrics analyzed in studies with people in the subacute phase after stroke focused on: motor function (LE-FMA) [241-245], gait endurance (6MWT) [69, 242, 244, 246, 247], and general mobility (TUG) $[242,244,246]$. The initial scores of the outcomes of interest that allowed for comparison between different control strategies were on average: LE-FMA = $18.87 \pm 3.75,6 \mathrm{MWT}=114.45 \pm 40.77 \mathrm{~m}$ and $\mathrm{TUG}$ $=29.42 \pm 10.2 \mathrm{~s}$. The number of participants and the training duration were on average $26.31 \pm 17.83$ and $672.72 \pm 453.89 \mathrm{~min}$, respectively.

Non-adaptive assistive control strategies that incorporated a threshold-based algorithm using EMG as the detection metric and control signal provided the highest improvements in all outcomes measures [241-243]. Importantly, this type of control showed similar or higher improvements with shorter training duration and higher grade of evidence (strong level) in LE-FMA (12.66\% improvement), 6MWT (69.59\%) and TUG (50.74\%), compared to the other control strategies implemented in other studies. However, the average number of participants ( 8 participants) was smaller than in other studies (25.5 participants), which reduces the impact of this result.

Nevertheless, as mentioned in the High-Level Control: Human-Robot Synchronization section, myoelectric control suffers from several technical limitations when employed in individuals with abnormal muscle activation patterns. Thus, it is possible that alternative detection metrics (e.g., based on lower-limb kinematics) and Mid-level control strategies (e.g., trajectory-tracking with/or compliant control) might produce greater improvements with shorter training time in subacute stroke participants [248-252].

Only one study combined two different control strategies separately on the same robotic treatment [244]. In particular, the authors combined EMG together with non-adaptive assistive control to a trajectory-tracking Mid-level control that used a threshold-based synchronization algorithm with ground reaction forces as detection metric in different sessions. When compared with other control strategies from other studies, the combination of the two control strategies in Watanabe et. al. [244] reached similar improvements with shorter training time and higher grade of evidence in LE-FMA (8.42 \%), 6MWT (60.39 $\%)$, and TUG (39.57\%).
Chronic Stroke

Studies on people in the chronic phase after stroke were the only ones that used all the metrics described in the Outcomes of interest for the clinical comparison subsection. The mean baseline values (i.e., baseline condition) were: $6 \mathrm{MWT}=197.03 \pm 58.53 \mathrm{~m}$ $[156,167,170,189,253-262], 10 \mathrm{MWT}=0.42 \pm 0.23 \mathrm{~m} / \mathrm{s}$ $[156,167,170,253,256,263-268], \mathrm{BBS}=44.00 \pm 6.94$ [156,167,170,189,253,254,258,260-264,269-271], TUG $=31.85 \pm 20.00 \mathrm{~s}[156,189,253,256,257,260,268-270]$, and LE-FMA $=37.26 \pm 53.80[156,167,169,170,189$, $255,257,258,260,267]$. The average number of participants per study and the mean training duration were $16.95 \pm 12.80$ and $1036.09 \pm 905.31 \mathrm{~min}$, respectively.

Results present a preeminence of adaptive over nonadaptive control strategies in studies with people in the chronic phase of stroke. Specifically, adaptive assistance, together with adaptive oscillators that use lower-limb kinematic information to synchronize the robot with the user's motion and with a trajectorytracking control as Mid-level control, achieved the best results. Robotic treatments using adaptive control strategies showed higher or similar improvements -i.e., improvement of $46.0 \%$ in $6 \mathrm{MWT}, 34.0 \%$ in 10MWT, $25.10 \%$ in LE-FMA, and $11.30 \%$ in BBS after the treatment- with shorter or similar training duration and higher grade of evidence, compared to other control strategies implemented in other studies $[167,169]$. Control strategies that implemented adaptive assistance in combination with trajectory tracking and compliant control showed the highest increase in TUG $(20.42 \%)$ and LE-FMA (27.76\%), with a strong grade of evidence [255-258, 267-270]. However, these studies also involved the longest training duration. Therefore, the superior improvement might be related not only to the control strategy employed, but also to the longer training duration (mean: $1148.3 \mathrm{~min}$ ) in comparison with the studies that used different control strategies and that also evaluated these metrics (mean: $743.5 \mathrm{~min}$ ).

Similar improvements in the 10MWT and BBS (28.82 \% and $12.39 \%$, respectively) were observed 920 when non-adaptive assistance controllers with a threshold ${ }_{\oplus 21}$ based approach using EMG as detection metric and ${ }_{922}$ control signal were employed [263-265]. However, the ${ }_{923}$ grade of evidence and the number of participants were $\quad{ }_{924}$ lower in comparison to the aforementioned studies that implemented adaptive controllers. Furthermore, this type of controller was the only one that had a negative effect on the TUG score $(-3.61 \%)$ [263, 264].

Only one study evaluated adaptive resistive control strategies in people in the chronic phase of stroke [261]. The authors reported an increase in 6MWT $(5.00 \%)$ and BBS (7.14 \%), which are similar to the ones reported for adaptive assistive control. Based on this, 
we advocate that more studies implementing resistive control strategies need to be carried out to provide stronger evidence on their clinical effectiveness.

\section{Discussion}

The main contribution of this systematic review is that it provides a classification of the control strategies implemented on lower-limb exoskeletons, analyzes the experimental methodology used in the robotic interventions, and compares the clinical effectiveness of the control strategies when used -together with the exoskeleton- as a gait rehabilitation tool for people with brain injuries. In the following subsections, we respond to the posed three research questions of this review.

\section{Which control strategies have been used on powered} lower limb exoskeletons for people with brain injuries? Regarding the implementation of High-level controllers, we found that adaptive assistive control strategies are the most widely implemented on lowerlimb exoskeletons for people with brain injuries. Although they were shown to be effective, most of the controllers included in this review did not adapt the assistance based on meaningful biomechanical metrics such as hip hiking or circumduction. Thus, it is an open question whether adaptive controllers that modulate the assistance based on biomechanical descriptors of the participants' current impairments, e.g., propulsion force and foot clearance, would potentially outperform current solutions. Comprehensive studies analyzing the effect of the exoskeleton control parameters on clinically meaningful biomechanical metrics might allow the development of adaptive control rules that directly tackle the main gait abnormalities of people with brain injuries [272,273].

As for human-robot synchronization, we found that threshold-based techniques, which rely on ground reaction force as detection metric, are extensively used. Only a few devices used adaptive oscillators to synchronize the motion of the exoskeleton with that of the user. Yet, adaptive oscillators seem to have a high potential for this specific population. As an interesting result, only one device included in this systematic review implemented stochastic methods for human-robot synchronization, despite their popularity in research and potential application in identification and classification of states and actions of the human-robot system. In recent years, novel approaches have been proposed that estimate biological joint torques using musculoskeletal modelling to control the action of the exoskeleton in a state-independent manner, i.e., with no need to detect gait events or different walking conditions, e.g., stair ascent and descent $[150,274,275]$. However, these control strategies still need further investigation to evaluate their potential clinical effectiveness on people with brain-injury.

For the Mid-level control, position trajectorytracking control was the most commonly used strategy, which was combined in some cases with compliant control to dynamically relate joint angles to forces or torques. We consider that this approach might be the most appropriate for devices that provide adaptive assistance, as it fosters the dynamic synergy between the user and the device. Only a few devices implemented myoelectric control, while none of them employed BCI to control lower-limb exoskeletons in this population. We attribute this shortage to the difficulty of developing generalized control laws that use EMG or EEG as control signals with people with brain injuries.

What are the experimental protocols and outcome metrics used for the clinical validation of robotic interventions?

We found a wide heterogeneity in the experimental protocols and the selection of the outcomes of interest to evaluate the robotic interventions. Walking speed was the preferred metric to evaluate the participants' initial impairment level and the effectiveness of the robotic treatment. Almost all studies included in this review focused on testing the exoskeletons on participants with stroke. Other types of brain injury represented a low portion of the reviewed studies. The variety in the experimental protocols and the reported performance metrics are the main factors which hinder the systematic comparison between the controllers' effectiveness.

What is the current clinical evidence on the effectiveness of the different control strategies?

Adaptive assistive control strategies that implemented a combination of trajectory-tracking and compliant control showed the highest clinical effectiveness for acute stroke. Non-adaptive assistive control strategies that followed a threshold-based algorithm with EMG as detection metric and control signal provided the highest improvements in the outcome measures of interest for subacute stroke. Finally, adaptive oscillators that used lower limb kinematic information to assist the motion of the user together with trajectorytracking as Mid-level control showed the highest improvements for chronic stroke. Note that these conclusions should be treated with the consideration that the number of studies for the clinical comparison of the control strategies was low. 
Limitations and future steps

Although the number of studies that evaluated the effectiveness of robotic-assisted gait rehabilitation has exponentially increased in the last decade, we still found critical limitations in the clinical comparison of the effectiveness of different control strategies. Only a few studies compared different control strategies on the same participants and using the same exoskeleton, hindering the possibility to extract clear conclusions regarding the clinical effectiveness of each control strategy for gait rehabilitation. In addition, spontaneous recovery [69] and compensation strategies probably contributed to increased scores on the outcome metrics, making it challenging to purely evaluate the effect of the different control strategies on functional recovery among different studies.

Despite the average level of evidence of the studies included in the clinical comparison is high, the number of studies for each family of control strategies is still small. The reduced number of studies might be a consequence of the regulatory framework for medical devices, which limits the opportunity of validating the technology at early stages of development. With current tight regulations, testing devices at a low Technology Readiness Level (TRL) is subject to the same requirements as those devices that are ready to be certified $[117,178,276]$. There is a lack of an ethical and regulatory framework that enables researchers to involve end-users in the co-creation and validation of early-stage prototypes to quickly make technology accessible to the users, while guaranteeing the well-being of patients and therapists.

\section{Conclusion}

Although remarkable efforts have been made into developing novel sophisticated motor-learning driven controllers to enhance gait rehabilitation, the majority of the reviewed studies only provided a general overview of the effect of the robotic controller on patients with brain injuries. Future research should evolve into structured and standardized studies that aim at finding the relation between control strategies and clinical outcome measures, controlling for the effects of participants' initial impairment level and training duration. Current limitations might be overcome when clinicians, researchers, industry, and regulatory bodies work together to solve this urgent societal problem.

Ethics approval and consent to participate

Not applicable.
Availability of data and materials

All data generated or analysed during this study are included in this published article and its supplementary information files.

Competing interests

The authors declare that they have no competing interests.

\section{Funding}

The present research was partly supported by grant No. 2020 FI_B 00331 funded by the Agency for Management of University and Research Grants (AGAUR) along with the Secretariat of Universities and Research of the Catalan Ministry of Business and Knowledge and the European Social Fund (ESF), by grant PTQ2018-010227 funded by the Spanish Ministry of Science and Innovation (MCI) - Agencia Estatal de Investigación (AEI), and by the Swiss National Science Foundation through the Grant PP00P2163800 and the Dutch Research Council (NWO) Talent Program VIDI TTW 2020.

\section{Authors' contributions}

JDMF performed the main review of literature, drafted and wrote the manuscript and collected the information to create the data sheets. JLP, LMC, EP and JMFL provided important content, structured the study, and were actively involved in the writing process of the manuscript. All authors read and approved the final manuscript.

\section{Acknowledgements}

The authors would like to thank Katlin Kreamer-Tonin (Product Manager at ABLE Human Motion, Barcelona, Spain) for proofreading the final version of the manuscript.

\section{Author details}

${ }^{1}$ Biomechanical Engineering Lab, Department of Mechanical Engineering and Research Centre for Biomedical Engineering, Universitat Politècnica de Catalunya, Diagonal 647, 08028, Barcelona, Spain. ${ }^{2}$ Institut de Recerca Sant Joan de Déu, Santa Rosa 39-57 08950 Esplugues de Llobregat, Spain. ${ }^{3}$ ABLE Human Motion, Diagonal 647, 08028, Barcelona, Spain.

${ }^{4}$ Roessingh Research and Development, Roessinghsbleekweg 33b 7522AH Enschede, Netherlands. ${ }^{5}$ Cognitive Robotics, Delft University of Technology, Mekelweg 2, 2628, Delft, Netherlands. ${ }^{6}$ Motor Learning and Neurorehabilitation Lab, ARTORG Center for Biomedical Engineering Research, University of Bern, Freiburgstrasse 3, 3010, Bern, Switzerland.

References

1. Cieza, A., Causey, K., Kamenov, K., Hanson, S.W., Chatterji, S., Vos, T.: Global estimates of the need for rehabilitation based on the global burden of disease study 2019: a systematic analysis for the global burden of disease study 2019. The Lancet 396(10267), 2006-2017 (2020)

2. Johnson, C.O., Nguyen, M., Roth, G.A., Nichols, E., Alam, T., Abate, D., Abd-Allah, F., Abdelalim, A., Abraha, H.N., Abu-Rmeileh, N.M., et al.: Global, regional, and national burden of stroke, 1990-2016: a systematic analysis for the global burden of disease study 2016. The Lancet Neurology 18(5), 439-458 (2019)

3. Johnson, W., Onuma, O., Owolabi, M., Sachdev, S.: Stroke: a global response is needed. Bulletin of the World Health Organization 94(9), 634 (2016)

4. McGuire, D.O., Tian, L.H., Yeargin-Allsopp, M., Dowling, N.F., Christensen, D.L.: Prevalence of cerebral palsy, intellectual disability, hearing loss, and blindness, national health interview survey, 2009-2016. Disability and health journal 12(3), 443-451 (2019)

5. Sellier, E., Platt, M.J., Andersen, G.L., Krägeloh-Mann, I., De La Cruz, J., Cans, C., of Cerebral Palsy Network, S., Van Bakel, M., Arnaud, C., Delobel, M., et al.: Decreasing prevalence in cerebral palsy: a multi-site european population-based study, 1980 to 2003. Developmental Medicine \& Child Neurology 58(1), 85-92 (2016)

6. Dewan, M.C., Rattani, A., Gupta, S., Baticulon, R.E., Hung, Y.-C., Punchak, M., Agrawal, A., Adeleye, A.O., Shrime, M.G., Rubiano, A.M., et al.: Estimating the global incidence of traumatic brain injury. Journal of neurosurgery 130(4), 1080-1097 (2018)

7. Crichton, S.L., Bray, B.D., McKevitt, C., Rudd, A.G., Wolfe, C.D. Patient outcomes up to 15 years after stroke: survival, disability, $\quad 1150$ quality of life, cognition and mental health. J Neurol Neurosurg 1151 Psychiatry 87(10), 1091-1098 (2016) 
8. Kelly-Hayes, M., Beiser, A., Kase, C.S., Scaramucci, A., D'Agostino, R.B., Wolf, P.A.: The influence of gender and age on disability following ischemic stroke: the framingham study. Journal of Stroke and Cerebrovascular Diseases 12(3), 119-126 (2003)

9. Jørgensen, H.S., Nakayama, H., Raaschou, H.O., Olsen, T.S.: Recovery of walking function in stroke patients: the copenhagen stroke study. Archives of physical medicine and rehabilitation 76(1), 27-32 (1995)

10. Winstein, C.J., Stein, J., Arena, R., Bates, B., Cherney, L.R., Cramer, S.C., Deruyter, F., Eng, J.J., Fisher, B., Harvey, R.L., et al.: Guidelines for adult stroke rehabilitation and recovery: a guideline for healthcare professionals from the american heart association/american stroke association. Stroke 47(6), 98-169 (2016)

11. Teasell, R., Viana, R.: Evidence-based benefit of rehabilitation after stroke. Textbook of Neural Repair and Rehabilitation; Cambridge University Press: Cambridge, UK; London, UK, 601-614 (2014)

12. Roelker, S.A., Bowden, M.G., Kautz, S.A., Neptune, R.R.: Paretic propulsion as a measure of walking performance and functional motor recovery post-stroke: a review. Gait \& posture (2018)

13. Lin, P.-Y., Yang, Y.-R., Cheng, S.-J., Wang, R.-Y.: The relation between ankle impairments and gait velocity and symmetry in people with stroke. Archives of physical medicine and rehabilitation 87(4), 562-568 (2006)

14. Murray, S.A., Ha, K.H., Hartigan, C., Goldfarb, M.: An assistive control approach for a lower-limb exoskeleton to facilitate recovery of walking following stroke. IEEE Transactions on Neural Systems and Rehabilitation Engineering 23(3), 441-449 (2014)

15. Wiszomirska, I., Błażkiewicz, M., Kaczmarczyk, K. Brzuszkiewicz-Kuźmicka, G., Wit, A.: Effect of drop foot on spatiotemporal, kinematic, and kinetic parameters during gait. Applied bionics and biomechanics 2017 (2017)

16. Woolley, S.M.: Characteristics of gait in hemiplegia. Topics in stroke rehabilitation 7(4), 1-18 (2001)

17. Bernhardt, J., Hayward, K.S., Kwakkel, G., Ward, N.S., Wolf, S.L., Borschmann, K., Krakauer, J.W., Boyd, L.A., Carmichael, S.T., Corbett, D., et al.: Agreed definitions and a shared vision for new standards in stroke recovery research: the stroke recovery and rehabilitation roundtable taskforce. International Journal of Stroke 12(5), 444-450 (2017)

18. Hebert, D., Lindsay, M.P., Mclntyre, A., Kirton, A., Rumney, P.G., Bagg, S., Bayley, M., Dowlatshahi, D., Dukelow, S., Garnhum, M., et al.: Canadian stroke best practice recommendations: stroke rehabilitation practice guidelines, update 2015. International Journal of Stroke 11(4), 459-484 (2016)

19. Teasell, R., Hussein, N.: Stroke Rehabilitation Clinician Handbook. Chapter 4. Motor Rehabilitation: Lower Extremity and Mobility (2016)

20. Teasell, R., Hussein, N.: Stroke Rehabilitation Clinician Handbook. Chapter 2. Brain Reorganization, Recovery, and Organized Care. 2016 (2016)

21. Schröder, J., Truijen, S., Van Criekinge, T., Saeys, W.: Feasibility and effectiveness of repetitive gait training early after stroke: A systematic review and meta-analysis. Journal of rehabilitation medicine 51(2), 78-88 (2019)

22. Kwah, L., Kwakkel, G., Veerbeek, J.: Prediction of Motor Recovery and Outcomes After Stroke. Stroke Rehabilitation, pp. 23-47. Elsevier, Netherlands (2018) doi:10.1016/b978-0-323-55381-0.00002-0

23. Langhorne, P., Bernhardt, J., Kwakkel, G.: Stroke rehabilitation. The Lancet 377(9778), 1693-1702 (2011)

24. Koenig, A., Omlin, X., Bergmann, J., Zimmerli, L., Bolliger, M., Müller, F., Riener, R.: Controlling patient participation during robot-assisted gait training. Journal of neuroengineering and rehabilitation 8(1), 1-12 (2011)

25. Kim, B., Deshpande, A.D.: An upper-body rehabilitation exoskeleton harmony with an anatomical shoulder mechanism: Design, modeling, control, and performance evaluation. The International Journal of Robotics Research 36(4), 414-435 (2017)

26. Fisher, B.E., Sullivan, K.J.: Activity-dependent factors affecting poststroke functional outcomes. Topics in stroke rehabilitation 8(3), 31-44 (2001)
27. Krakauer, J.W.: Motor learning: its relevance to stroke recovery and neurorehabilitation. Current opinion in neurology 19(1), 84-90 (2006)

28. Schmidt, R.A., Lee, T.D., Winstein, C., Wulf, G., Zelaznik, H.N. Human kinetics (2018)

29. Mehrholz, J., Thomas, S., Kugler, J., Pohl, M., Elsner, B.: Electromechanical-assisted training for walking after stroke. Cochrane database of systematic reviews (10) (2020)

30. Goffredo, M., lacovelli, C., Russo, E., Pournajaf, S., Di Blasi, C., Galafate, D., Pellicciari, L., Agosti, M., Filoni, S., Aprile, I., et al.: Stroke gait rehabilitation: A comparison of end-effector, overground exoskeleton, and conventional gait training. Applied Sciences 9(13), 2627 (2019)

31. Tedla, J.S., Dixit, S., Gular, K., Abohashrh, M.: Robotic-assisted gait training effect on function and gait speed in subacute and chronic stroke population: A systematic review and meta-analysis of randomized controlled trials. European neurology, 1-9 (2019)

32. Moucheboeuf, G., Griffier, R., Gasq, D., Glize, B., Bouyer, L., Dehail, P., Cassoudesalle, H.: Effects of robotic gait training after stroke: a meta-analysis. Annals of Physical and Rehabilitation Medicine (2020)

33. Sczesny-Kaiser, M., Trost, R., Aach, M., Schildhauer, T.A., Schwenkreis, P., Tegenthoff, M.: A randomized and controlled crossover study investigating the improvement of walking and posture functions in chronic stroke patients using hal exoskeleton-the halestro study (hal-exoskeleton stroke study). Frontiers in neuroscience 13, 259 (2019)

34. Roth, E.J., Merbitz, C., Mroczek, K., Dugan, S.A., Suh, W.W Hemiplegic gait: Relationships between walking speed and other temporal parameters1. American journal of physical medicine \& rehabilitation 76(2), 128-133 (1997)

35. Trushkova, N., Cochran, O., Ermolina, N., Zelano, G.: Is training with a focus on motor learning effective in improving body coordination in chronic post stroke patients? Journal of the Neurological Sciences 429, 118583 (2021)

36. Marchal-Crespo, L., Riener, R.: Robot-assisted gait training, pp 227-240. Elsevier (2018)

37. Marks, D., Schweinfurther, R., Dewor, A., Huster, T., Paredes, L.P., Zutter, D., Möller, J.C.: The andago for overground gait training in patients with gait disorders after stroke-results from a usability study. Physiother Res Rep 2, 1-8 (2019)

38. Zhang, X., Yue, Z., Wang, J.: Robotics in lower-limb rehabilitation after stroke. Behavioural neurology 2017 (2017)

39. Chrif, F., Nef, T., Lungarella, M., Dravid, R., Hunt, K.J.: Control design for a lower-limb paediatric therapy device using linear motor technology. Biomedical signal processing and control 38, 119-127 (2017)

40. Rodríguez-Fernández, A., Lobo-Prat, J., Font-Llagunes, J.M.: Systematic review on wearable lower-limb exoskeletons for gait training in neuromuscular impairments. Journal of neuroengineering and rehabilitation 18(1), 1-21 (2021)

41. Baud, R., Manzoori, A., ljspeert, A.J., Bouri, M.: Review of control strategies for lower-limb exoskeletons to assist gait. Journal of neuroengineering and rehabilitation 18 (2021). doi:10.1186/s12984-021-00906-3

42. Li, W.-Z., Cao, G.-Z., Zhu, A.-B.: Review on control strategies for lower limb rehabilitation exoskeletons. IEEE Access (2021)

43. Young, A.J., Ferris, D.P.: State of the art and future directions for lower limb robotic exoskeletons. IEEE Transactions on Neural Systems and Rehabilitation Engineering 25(2), 171-182 (2016)

44. Meng, W., Liu, Q., Zhou, Z., Ai, Q., Sheng, B., Xie, S.S.: Recent development of mechanisms and control strategies for robot-assisted lower limb rehabilitation. Mechatronics 31, 132-145 (2015)

45. Shi, D., Zhang, W., Zhang, W., Ding, X.: A review on lower limb rehabilitation exoskeleton robots. Chinese Journal of Mechanical Engineering 32(1), 1-11 (2019)

46. Contreras-Vidal, J.L., Bhagat, N.A Brantley, J., Cruz-Garza, J.G. He, Y., Manley, Q., Nakagome, S., Nathan, K., Tan, S.H., Zhu, F., et 1290 al.: Powered exoskeletons for bipedal locomotion after spinal cord 1291 injury. Journal of neural engineering 13(3), 031001 (2016)

47. Huo, W., Mohammed, S., Moreno, J.C., Amirat, Y.: Lower limb wearable robots for assistance and rehabilitation: A state of the art. 
IEEE systems Journal 10(3), 1068-1081 (2014)

48. Esquenazi, A., Talaty, M.: Robotics for lower limb rehabilitation. Physical Medicine and Rehabilitation Clinics 30(2), 385-397 (2019)

49. Chen, B., Ma, H., Qin, L.-Y., Gao, F., Chan, K.-M., Law, S.-W., Qin, L., Liao, W.-H.: Recent developments and challenges of lower extremity exoskeletons. Journal of Orthopaedic Translation 5, 26-37 (2016)

50. del Carmen Sanchez-Villamañan, M., Gonzalez-Vargas, J., Torricelli, D., Moreno, J.C., Pons, J.L.: Compliant lower limb exoskeletons: a comprehensive review on mechanical design principles. Journal of neuroengineering and rehabilitation 16(1), 55 (2019)

51. Morone, G., Paolucci, S., Cherubini, A., De Angelis, D., Venturiero, V., Coiro, P., losa, M.: Robot-assisted gait training for stroke patients: current state of the art and perspectives of robotics. Neuropsychiatric disease and treatment 13, 1303 (2017)

52. Weber, L.M., Stein, J.: The use of robots in stroke rehabilitation: A narrative review. NeuroRehabilitation 43(1), 99-110 (2018)

53. Louie, D.R., Eng, J.J.: Powered robotic exoskeletons in post-stroke rehabilitation of gait: a scoping review. Journal of neuroengineering and rehabilitation 13(1), 53 (2016)

54. Marchal-Crespo, L., Reinkensmeyer, D.J.: Review of control strategies for robotic movement training after neurologic injury. Journal of neuroengineering and rehabilitation 6(1), 20 (2009)

55. Tucker, M.R., Olivier, J., Pagel, A., Bleuler, H., Bouri, M., Lambercy, O., del R Millán, J., Riener, R., Vallery, H., Gassert, R.: Control strategies for active lower extremity prosthetics and orthotics: a review. Journal of neuroengineering and rehabilitation 12(1), 1 (2015)

56. Yan, T., Cempini, M., Oddo, C.M., Vitiello, N.: Review of assistive strategies in powered lower-limb orthoses and exoskeletons. Robotics and Autonomous Systems 64, 120-136 (2015)

57. Chen, B., Zi, B., Qin, L., Pan, Q.: State-of-the-art research in robotic hip exoskeletons: A general review. Journal of Orthopaedic Translation (2019)

58. Li, M., Xu, G., Xie, J., Chen, C.: A review: Motor rehabilitation after stroke with control based on human intent. Proceedings of the Institution of Mechanical Engineers, Part $\mathrm{H}$ : Journal of Engineering in Medicine 232(4), 344-360 (2018)

59. Shi, B., Chen, X., Yue, Z., Yin, S., Weng, Q., Zhang, X., Wang, J., Wen, W.: Wearable ankle robots in post-stroke rehabilitation of gait: A systematic review. Frontiers in neurorobotics 13, 63 (2019)

60. Hobbs, B., Artemiadis, P.: A review of robot-assisted lower-limb stroke therapy: unexplored paths and future directions in gait rehabilitation. Frontiers in neurorobotics 14 (2020)

61. Xiloyannis, M., Alicea, R., Georgarakis, A.-M., Haufe, F.L., Wolf, P., Masia, L., Riener, R.: Soft robotic suits: State of the art, core technologies, and open challenges. IEEE Transactions on Robotics (2021)

62. Madhav, M.S., Cowan, N.J.: The synergy between neuroscience and control theory: the nervous system as inspiration for hard control challenges. Annual Review of Control, Robotics, and Autonomous Systems 3, 243-267 (2020)

63. Palisano, R.J., Rosenbaum, P., Bartlett, D., Livingston, M.H.: Content validity of the expanded and revised gross motor function classification system. Developmental Medicine \& Child Neurology 50(10), 744-750 (2008)

64. Nilsson, A., Vreede, K.S., Häglund, V., Kawamoto, H., Sankai, Y., Borg, J.: Gait training early after stroke with a new exoskeleton-the hybrid assistive limb: a study of safety and feasibility. Journal of neuroengineering and rehabilitation 11(1), 1-11 (2014)

65. Van Nunen, M.P.M., Gerrits, K.H.L., Konijnenbelt, M., Janssen, T.W.J., De Haan, A.: Recovery of walking ability using a robotic device in subacute stroke patients: A randomized controlled study. Disability and Rehabilitation: Assistive Technology 10(2), 141-148 (2015). doi:10.3109/17483107.2013.873489

66. Wall, A., Borg, J., Vreede, K., Palmcrantz, S.: A randomized controlled study incorporating an electromechanical gait machine, the Hybrid Assistive Limb, in gait training of patients with severe limitations in walking in the subacute phase after stroke. PloS one 15(2), 0229707 (2020). doi:10.1371/journal.pone.0229707

67. Leon, D., Cortes, M., Elder, J., Kumru, H., Laxe, S., Edwards, D.J.,
Tormos, J.M., Bernabeu, M., Pascual-Leone, A.: TDCS does not enhance the effects of robot-assisted gait training in patients with subacute stroke. Restorative Neurology and Neuroscience 35(4), 377-384 (2017). doi:10.3233/RNN-170734

68. Husemann, B., Müller, F., Krewer, C., Heller, S., Koenig, E.: Effects of locomotion training with assistance of a robot-driven gait orthosis in hemiparetic patients after stroke: a randomized controlled pilot study. Stroke 38(2), 349-354 (2007). doi:10.1161/01.STR.0000254607.48765.cb

69. Molteni, F., Gasperini, G., Gaffuri, M., Colombo, M., Giovanzana, C. Lorenzon, C., Farina, N., Cannaviello, G., Scarano, S., Proserpio, D., Liberali, D., Guanziroli, E.: Wearable robotic exoskeleton for overground gait training in sub-acute and chronic hemiparetic stroke patients: preliminary results. European journal of physical and rehabilitation medicine 53(5), 676-684 (2017). doi:10.23736/S1973-9087.17.04591-9

70. Haynes, R.B., Sackett, D.L., Richardson, W.S., Rosenberg, W., Langley, G.R.: Evidence-based medicine: How to practice \& teach ebm. Canadian Medical Association. Journal 157(6), 788 (1997)

71. Guyatt, G.H., Rennie, D.: Users' guides to the medical literature. Jama 270(17), 2096-2097 (1993)

72. Sullivan, J.E., Crowner, B.E., Kluding, P.M., Nichols, D., Rose, D.K., Yoshida, R., Pinto Zipp, G.: Outcome measures for individuals with stroke: process and recommendations from the american physical therapy association neurology section task force. Physical therapy 93(10), 1383-1396 (2013)

73. Bushnell, C., Bettger, J.P., Cockroft, K.M., Cramer, S.C., Edelen, M.O., Hanley, D., Katzan, I.L., Mattke, S., Nilsen, D.M., Piquado, T., et al.: Chronic stroke outcome measures for motor function intervention trials: expert panel recommendations. Circulation: Cardiovascular Quality and Outcomes 8(6_suppl_3), 163-169 (2015)

74. Oeffinger, D., Bagley, A., Rogers, S., Gorton, G., Kryscio, R., Abel, M., Damiano, D., Barnes, D., Tylkowski, C.: Outcome tools used for ambulatory children with cerebral palsy: responsiveness and minimum clinically important differences. Developmental Medicine \& Child Neurology 50(12), 918-925 (2008)

75. Debuse, D., Brace, H.: Outcome measures of activity for children with cerebral palsy: a systematic review. Pediatric Physical Therapy 23(3), 221-231 (2011)

76. Knox, V., Vuoskoski, P., Mandy, A.: Use of outcome measures in children with severe cerebral palsy: A survey of uk physiotherapists. Physiotherapy Research International 24(4), 1786 (2019)

77. Ferre-Fernández, M., Murcia-González, M.A., Espinosa, M.D.B., Ríos-Díaz, J.: Measures of motor and functional skills for children with cerebral palsy: A systematic review. Pediatric Physical Therapy 32(1), 12-25 (2020)

78. Vargus-Adams, J.N.: Outcome assessment and function in cerebral palsy. Physical medicine and rehabilitation clinics of North America 31(1), 131-141 (2019)

79. Proietti, T., Crocher, V., Roby-Brami, A., Jarrasse, N.: Upper-limb robotic exoskeletons for neurorehabilitation: a review on control strategies. IEEE reviews in biomedical engineering 9, 4-14 (2016)

80. Basteris, A., Nijenhuis, S.M., Stienen, A.H., Buurke, J.H., Prange, G.B., Amirabdollahian, F.: Training modalities in robot-mediated upper limb rehabilitation in stroke: a framework for classification based on a systematic review. Journal of neuroengineering and rehabilitation 11(1), 1-15 (2014)

81. Basalp, E., Wolf, P., Marchal-Crespo, L.: Haptic training: Which types facilitate (re) learning of which motor task and for whom answers by a review. IEEE Transactions on Haptics (2021)

82. Shepherd, M.K., Rouse, E.J.: Design and validation of a torque-controllable knee exoskeleton for sit-to-stand assistance. IEEE/ASME Transactions on Mechatronics 22(4), 1695-1704 (2017)

83. Lerner, Z.F., Damiano, D.L., Bulea, T.C.: A robotic exoskeleton to treat crouch gait from cerebral palsy: Initial kinematic and neuromuscular evaluation. In: 2016 38th Annual International Conference of the IEEE Engineering in Medicine and Biology Society (EMBC), pp. 2214-2217 (2016). IEEE

84. Thalman, C.M., Hertzell, T., Lee, H.: Toward a soft robotic ankle-foot orthosis (sr-afo) exosuit for human locomotion: Preliminary results in late stance plantarflexion assistance. In: 2020 
3rd IEEE International Conference on Soft Robotics (RoboSoft), pp. 801-807 (2020). IEEE

85. Rossini, P.M., Dal Forno, G.: Integrated technology for evaluation of brain function and neural plasticity. Physical Medicine and Rehabilitation Clinics 15(1), 263-306 (2004)

86. Crespo, L.M., Reinkensmeyer, D.J.: Effect of robotic guidance on motor learning of a timing task. In: 2008 2nd IEEE RAS \& EMBS International Conference on Biomedical Robotics and Biomechatronics, pp. 199-204 (2008). IEEE

87. Harkema, S.J.: Neural plasticity after human spinal cord injury: application of locomotor training to the rehabilitation of walking. The Neuroscientist 7(5), 455-468 (2001)

88. Hesse, S., Kuhlmann, H., Wilk, J., Tomelleri, C., Kirker, S.G.: A new electromechanical trainer for sensorimotor rehabilitation of paralysed fingers: a case series in chronic and acute stroke patients. Journal of neuroengineering and rehabilitation 5(1), 1-6 (2008)

89. Reinkensmeyer, D.J., Kahn, L.E., Averbuch, M., McKenna-Cole, A., Schmit, B.D., Rymer, W.Z.: Understanding and treating arm movement impairment after chronic brain injury: progress with the arm guide. Journal of rehabilitation research and development 37(6), 653-662 (2014)

90. Cramer, S.C., Sur, M., Dobkin, B.H., O'brien, C., Sanger, T.D., Trojanowski, J.Q., Rumsey, J.M., Hicks, R., Cameron, J., Chen, D., et al:: Harnessing neuroplasticity for clinical applications. Brain 134(6), 1591-1609 (2011)

91. Escalona, M.J., Bourbonnais, D., Goyette, M., Duclos, C., Gagnon, D.H.: Wearable exoskeleton control modes selected during overground walking affect muscle synergies in adults with a chronic incomplete spinal cord injury. Spinal Cord Series and Cases 6(1), 1-9 (2020)

92. Conner, B.C., Luque, J., Lerner, Z.F.: Adaptive ankle resistance from a wearable robotic device to improve muscle recruitment in cerebral palsy. Annals of Biomedical Engineering, 1-13 (2020)

93. Wei, Y., Patton, J., Bajaj, P., Scheidt, R.: A real-time haptic/graphic demonstration of how error augmentation can enhance learning. In Proceedings of the 2005 IEEE International Conference on Robotics and Automation, pp. 4406-4411 (2005). IEEE

94. Blanchette, A.K., Noël, M., Richards, C.L., Nadeau, S., Bouyer, L.J.: Modifications in ankle dorsiflexor activation by applying a torque perturbation during walking in persons post-stroke: A case series. Journal of neuroengineering and rehabilitation 11(1) (2014). doi:10.1186/1743-0003-11-98

95. Veldema, J., Jansen, P.: Resistance training in stroke rehabilitation: systematic review and meta-analysis. Clinical Rehabilitation 34(9), 1173-1197 (2020)

96. Ouellette, M.M., LeBrasseur, N.K., Bean, J.F., Phillips, E., Stein, J., Frontera, W.R., Fielding, R.A.: High-intensity resistance training improves muscle strength, self-reported function, and disability in long-term stroke survivors. Stroke 35(6), 1404-1409 (2004)

97. Lamberti, N., Straudi, S., Malagoni, A.M., Argirò, M., Felisatti, M., Nardini, E., Zambon, C., Basaglia, N., Manfredini, F.: Effects of low-intensity endurance and resistance training on mobility in chronic stroke survivors: a pilot randomized controlled study. European journal of physical and rehabilitation medicine 53(2), 228-239 (2016)

98. Li, Y., Lamontagne, A., et al.: The effects of error-augmentation versus error-reduction paradigms in robotic therapy to enhance upper extremity performance and recovery post-stroke: a systematic review. Journal of neuroengineering and rehabilitation 15(1), 1-25 (2018)

99. Schmidt, R.A., Young, D.E., Swinnen, S., Shapiro, D.C.: Summary knowledge of results for skill acquisition: Support for the guidance hypothesis. Journal of Experimental Psychology: Learning, Memory, and Cognition 15(2), 352 (1989)

100. Poggensee, K.L., Collins, S.H.: How adaptation, training, and customization contribute to benefits from exoskeleton assistance. bioRxiv (2021)

101. Lv, G., Zhu, H., Gregg, R.D.: On the Design and Control of Highly Backdrivable Lower-Limb Exoskeletons: A Discussion of Past and Ongoing Work. IEEE Control Systems Magazine 38(6), 88-113 (2018). doi:10.1109/MCS.2018.2866605

102. Murray, S.A., Ha, K.H., Hartigan, C., Goldfarb, M.: An assistive control approach for a lower-limb exoskeleton to facilitate recovery of walking following stroke. IEEE Transactions on Neural Systems and
Rehabilitation Engineering 23(3), 441-449 (2015). doi:10.1109/TNSRE.2014.2346193

103. Lotze, M., Braun, C., Birbaumer, N., Anders, S., Cohen, L.G.: Motor learning elicited by voluntary drive. Brain 126(4), 866-872 (2003)

104. Oyake, K., Suzuki, M., Otaka, Y., Tanaka, S.: Motivational strategies for stroke rehabilitation: a descriptive cross-sectional study. Frontiers in neurology 11, 553 (2020)

105. Martinez, A., Lawson, B., Goldfarb, M.: A Velocity-Based Flow Field Control Approach for Reshaping Movement of Stroke-Impaired Individuals with a Lower-Limb Exoskeleton. Conference proceedings : 2018 Annual International Conference of the IEEE Engineering in Medicine and Biology Society. IEEE Engineering in Medicine and Biology Society. Annual Conference 2018, 2797-2800 (2018). doi:10.1109/EMBC.2018.8512807

106. Puyuelo-Quintana, G., Cano-de-la-Cuerda, R., Plaza-Flores, A., Garces-Castellote, E., Sanz-Merodio, D., Goni-Arana, A., Marin-Ojea, J., Garcia-Armada, E.: A new lower limb portable exoskeleton for gait assistance in neurological patients: a proof of concept study. Journal of neuroengineering and rehabilitation 17(1) (2020). doi:10.1186/s12984-020-00690-6

107. McCain, E.M., Dick, T.J.M., Giest, T.N., Nuckols, R.W., Lewek, M.D., Saul, K.R., Sawicki, G.S.: Mechanics and energetics of post-stroke walking aided by a powered ankle exoskeleton with speed-adaptive myoelectric control. Journal of neuroengineering and rehabilitation 16 (2019). doi:10.1186/s12984-019-0523-y

108. Mizukami, N., Takeuchi, S., Tetsuya, M., Tsukahara, A., Yoshida, K., Matsushima, A., Maruyama, Y., Tako, K., Hashimoto, M.: Effect of the synchronization-based control of a wearable robot having a non-exoskeletal structure on the hemiplegic gait of stroke patients. IEEE Transactions on Neural Systems and Rehabilitation Engineering 26(5), 1011-1016 (2018). doi:10.1109/TNSRE.2018.2817647

109. Meuleman, J., van Asseldonk, E., van Oort, G., Rietman, H., van der Kooij, H.: LOPES II-Design and Evaluation of an Admittance Controlled Gait Training Robot With Shadow-Leg Approach. IEEE Transactions on Neural Systems and Rehabilitation Engineering 24(3), 352-363 (2016). doi:10.1109/TNSRE.2015.2511448

110. Blaya, J.A., Herr, H.: Adaptive Control of a Variable-Impedance Ankle-Foot Orthosis to Assist Drop-Foot Gait. IEEE Transactions on Neural Systems and Rehabilitation Engineering 12(1), 24-31 (2004). doi:10.1109/TNSRE.2003.823266

111. Cecilia Villa-Parra, A., Lima, J., Delisle-Rodriguez, D., Vargas-Valencia, L., Frizera-Neto, A., Bastos, T.: Assessment of an Assistive Control Approach Applied in an Active Knee Orthosis Plus Walker for Post-Stroke Gait Rehabilitation. SENSORS 20(9) (2020). doi: $10.3390 /$ s20092452

112. Hassan, M., Kadone, H., Ueno, T., Hada, Y., Sankai, Y., Suzuki, K.: Feasibility of Synergy-Based Exoskeleton Robot Control in Hemiplegia. IEEE Transactions on Neural Systems and Rehabilitation Engineering 26(6), 1233-1242 (2018). doi:10.1109/TNSRE.2018.2832657

113. Zhu, H., Nesler, C., Divekar, N., Peddinti, V., Gregg, R.: Design principles for compact, backdrivable actuation in partial-assist powered knee orthoses. IEEE/ASME Transactions on Mechatronics (2021)

114. Kawamoto, H., Taal, S., Niniss, H., Hayashi, T., Kamibayashi, K., Eguchi, K., Sankai, Y.: Voluntary motion support control of Robot Suit HAL triggered by bioelectrical signal for hemiplegia. Conference proceedings : 2010 Annual International Conference of the IEEE Engineering in Medicine and Biology Society. IEEE Engineering in Medicine and Biology Society. Annual Conference 2010, 462-466 (2010). doi:10.1109/IEMBS.2010.5626191

115. Gui, K., Liu, H., Zhang, D.: Toward Multimodal Human-Robot Interaction to Enhance Active Participation of Users in Gait Rehabilitation. IEEE Transactions on Neural Systems and Rehabilitation Engineering 25(11), 2054-2066 (2017). doi:10.1109/TNSRE.2017.2703586

116. Grimmer, M., Schmidt, K., Duarte, J.E., Neuner, L., Koginov, G., Riener, R.: Stance and swing detection based on the angular velocity of lower limb segments during walking. Frontiers in neurorobotics 13, 57 (2019)

117. He, Y., Eguren, D., Luu, T.P., Contreras-Vidal, J.L.: Risk 
management and regulations for lower limb medical exoskeletons: a review. Medical devices (Auckland, NZ) 10, 89 (2017)

118. Alaoui, O.M., Expert, F., Morel, G., Jarrassé, N.: Using generic upper-body movement strategies in a free walking setting to detect gait initiation intention in a lower-limb exoskeleton. IEEE Transactions on Medical Robotics and Bionics 2(2), 236-247 (2020)

119. Chen, G., Qi, P., Guo, Z., Yu, H.: Gait-event-based synchronization method for gait rehabilitation robots via a bioinspired adaptive oscillator. IEEE Transactions on Biomedical Engineering 64(6), 1345-1356 (2016)

120. Miyake, T., Kobayashi, Y., Fujie, M.G., Sugano, S.: Timing of intermittent torque control with wire-driven gait training robot lifting toe trajectory for trip avoidance. In: 2017 International Conference on Rehabilitation Robotics (ICORR), pp. 320-325 (2017). IEEE

121. Nomura, S., Takahashi, Y., Sahashi, K., Murai, S., Kawai, M., Taniai, Y., Naniwa, T.: Power assist control based on human motion estimation using motion sensors for powered exoskeleton without binding legs. Applied Sciences 9(1), 164 (2019)

122. Gurriet, T., Tucker, M., Duburcq, A., Boeris, G., Ames, A.D.: Towards variable assistance for lower body exoskeletons. IEEE Robotics and Automation Letters 5(1), 266-273 (2019)

123. Laschowski, B., McNally, W., Wong, A., McPhee, J.: Environment classification for robotic leg prostheses and exoskeletons using deep convolutional neural networks. bioRxiv (2021)

124. Aguirre-Ollinger, G., Narayan, A., Yu, H.: Phase-synchronized assistive torque control for the correction of kinematic anomalies in the gait cycle. IEEE Transactions on Neural Systems and Rehabilitation Engineering 27(11), 2305-2314 (2019)

125. Marder, E., Bucher, D.: Central pattern generators and the control of rhythmic movements. Current biology 11(23), 986-996 (2001)

126. Aguirre-Ollinger, G.: Exoskeleton control for lower-extremity assistance based on adaptive frequency oscillators: Adaptation of muscle activation and movement frequency. Proceedings of the Institution of Mechanical Engineers, Part $\mathrm{H}$ : Journal of Engineering in Medicine 229(1), 52-68 (2015)

127. De La Fuente, J., Subramanian, S.C., Sugar, T.G., Redkar, S.: A robust phase oscillator design for wearable robotic systems. Robotics and Autonomous Systems 128, 103514 (2020)

128. Ronsse, R., Lenzi, T., Vitiello, N., Koopman, B., Van Asseldonk, E., De Rossi, S.M.M., Van Den Kieboom, J., Van Der Kooij, H., Carrozza, M.C., ljspeert, A.J.: Oscillator-based assistance of cyclical movements: model-based and model-free approaches. Medical \& biological engineering \& computing 49(10), 1173 (2011)

129. Fricke, S.S., Bayón, C., Der Kooij, H.V., F. Van Asseldonk, E.H.: Automatic versus manual tuning of robot-assisted gait training in people with neurological disorders. Journal of neuroengineering and rehabilitation 17(1) (2020). doi:10.1186/s12984-019-0630-9

130. Atashzar, S.F., Shahbazi, M., Patel, R.V.: Haptics-enabled interactive neurorehabilitation mechatronics: classification, functionality, challenges and ongoing research. Mechatronics 57, 1-19 (2019)

131. Gandolla, M., Guanziroli, E., D'Angelo, A., Cannaviello, G., Molteni, F., Pedrocchi, A.: Automatic setting procedure for exoskeleton-assisted overground gait: Proof of concept on stroke population. Frontiers in Neurorobotics 12(MAR), 1-11 (2018). doi:10.3389/fnbot.2018.00010

132. Huang, C., Li, Y., Yao, X.: A survey of automatic parameter tuning methods for metaheuristics. IEEE transactions on evolutionary computation 24(2), 201-216 (2019)

133. Schicketmueller, A., Rose, G., Hofmann, M.: Feasibility of a sensor-based gait event detection algorithm for triggering functional electrical stimulation during robot-assisted gait training. Sensors 19(21), 4804 (2019)

134. Seel, T., Landgraf, L., Schauer, T.: Online gait phase detection with automatic adaption to gait velocity changes using accelerometers and gyroscopes. Biomed. Tech 59, 795-798 (2014)

135. Muller, P., Steel, T., Schauer, T.: Experimental evaluation of a novel inertial sensor based realtime gait phase detection algorithm. In: Proceedings of the Technically Assisted Rehabilitation Conference (2015)

136. Franks, P.W., Bryan, G.M., Martin, R.M., Reyes, R., Collins, S.H.: Comparing optimized exoskeleton assistance of the hip, knee, and ankle in single and multi-joint configurations. bioRxiv (2021)

137. Lora-Millan, J.S., Sanchez-Cuesta, F.J., Romero, J.P., Moreno, J.C., Rocon, E.: A unilateral robotic knee exoskeleton to assess the role of natural gait assistance in hemiparetic patients (2021)

138. Emken, J.L., Harkema, S.J., Beres-Jones, J.A., Ferreira, C.K., Reinkensmeyer, D.J.: Feasibility of manual teach-and-replay and continuous impedance shaping for robotic locomotor training following spinal cord injury. IEEE Transactions on Biomedical Engineering 55(1), 322-334 (2007)

139. Manchola, M.D.S., Mayag, L.J.A., Munera, M., García, C.A.C.: Impedance-based backdrivability recovery of a lower-limb exoskeleton for knee rehabilitation. In: 2019 IEEE 4th Colombian Conference on Automatic Control (CCAC), pp. 1-6 (2019). IEEE

140. Gordleeva, S.Y., Lobov, S.A., Grigorev, N.A., Savosenkov, A.O., Shamshin, M.O., Lukoyanov, M.V., Khoruzhko, M.A., Kazantsev, V.B.: Real-time eeg-emg human-machine interface-based control system for a lower-limb exoskeleton. IEEE Access 8, 84070-84081 (2020)

141. Gordon, K.E., Ferris, D.P.: Learning to walk with a robotic ankle exoskeleton. Journal of biomechanics 40(12), 2636-2644 (2007)

142. Tan, C.K., Kadone, H., Watanabe, H., Marushima, A., Yamazaki, M., Sankai, Y., Suzuki, K.: Lateral symmetry of synergies in Lower Limb muscles of acute post-stroke patients after robotic intervention. Frontiers in Neuroscience 12(APR) (2018). doi:10.3389/fnins.2018.00276

143. Benabid, A.L., Costecalde, T., Eliseyev, A., Charvet, G., Verney, A., Karakas, S., Foerster, M., Lambert, A., Morinière, B., Abroug, N., et al.: An exoskeleton controlled by an epidural wireless brain-machine interface in a tetraplegic patient: a proof-of-concept demonstration. The Lancet Neurology 18(12), 1112-1122 (2019)

144. Xu, R., Jiang, N., Mrachacz-Kersting, N., Lin, C., Prieto, G.A., Moreno, J.C., Pons, J.L., Dremstrup, K., Farina, D.: A closed-loop brain-computer interface triggering an active ankle-foot orthosis for inducing cortical neural plasticity. IEEE Transactions on Biomedical Engineering 61(7), 2092-2101 (2014)

145. Calanca, A., Muradore, R., Fiorini, P.: A review of algorithms for compliant control of stiff and fixed-compliance robots. IEEE/ASME Transactions on Mechatronics 21(2), 613-624 (2015)

146. Schumacher, M., Wojtusch, J., Beckerle, P., von Stryk, O.: An introductory review of active compliant control. Robotics and Autonomous Systems 119, 185-200 (2019)

147. Nagarajan, U., Aguirre-Ollinger, G., Goswami, A.: Integral admittance shaping: A unified framework for active exoskeleton control. Robotics and Autonomous Systems 75, 310-324 (2016)

148. Liang, W.: Mechanical design and control strategy for hip joint power assisting. Journal of healthcare engineering 2018 (2018)

149. Aguirre-Ollinger, G., Colgate, J.E., Peshkin, M.A., Goswami, A.: Active-impedance control of a lower-limb assistive exoskeleton. In: 2007 IEEE 10th International Conference on Rehabilitation Robotics, pp. 188-195 (2007). IEEE

150. Lotti, N., Xiloyannis, M., Durandau, G., Galofaro, E., Sanguineti, V., Masia, L., Sartori, M.: Adaptive model-based myoelectric control for a soft wearable arm exosuit: A new generation of wearable robot control. IEEE Robotics \& Automation Magazine 27(1), 43-53 (2020)

151. Adams, R.J., Hannaford, B.: Stable haptic interaction with virtual environments. IEEE Transactions on robotics and Automation 15(3), 465-474 (1999)

152. Hogan, N.: Impedance control: An approach to manipulation: Part i-theory (1985)

153. Koopman, B., Van Asseldonk, E.H.F., Van Der Kooij, H.: Selective control of gait subtasks in robotic gait training: Foot clearance support in stroke survivors with a powered exoskeleton. Journal of neuroengineering and rehabilitation 10(1) (2013). doi:10.1186/1743-0003-10-3

154. Gasparri, G.M., Luque, J., Lerner, Z.F.: Proportional Joint-Moment Control for Instantaneously Adaptive Ankle Exoskeleton Assistance. IEEE Transactions on Neural Systems and Rehabilitation Engineering 27(4), 751-759 (2019). doi:10.1109/TNSRE.2019.2905979

155. Siviy, C., Bae, J., Baker, L., Porciuncula, F., Baker, T., Ellis, T.D., Awad, L.N., Walsh, C.J.: Offline Assistance Optimization of a Soft Exosuit for Augmenting Ankle Power of Stroke Survivors during 
Walking. IEEE Robotics and Automation Letters 5(2), 828-835 (2020). doi:10.1109/LRA.2020.2965072

156. Yeung, L.F., Ockenfeld, C., Pang, M.K., Wai, H.W., Soo, O.Y., Li, S.W., Tong, K.Y.: Randomized controlled trial of robot-assisted gait training with dorsiflexion assistance on chronic stroke patients wearing ankle-foot-orthosis. Journal of neuroengineering and rehabilitation 15(1) (2018). doi:10.1186/s12984-018-0394-7

157. Conner, B.C., Luque, J., Lerner, Z.F.: Adaptive Ankle Resistance from a Wearable Robotic Device to Improve Muscle Recruitment in Cerebral Palsy. Annals of Biomedical Engineering 48(4), 1309-1321 (2020). doi:10.1007/s10439-020-02454-8

158. Yen, S.-C., Schmit, B.D., Wu, M.: Using swing resistance and assistance to improve gait symmetry in individuals post-stroke. Human Movement Science 42, 212-224 (2015). doi:10.1016/j.humov.2015.05.010

159. Asin-Prieto, G., Martinez-Exposito, A., Barroso, F.O., Urendes, E.J., Gonzalez-Vargas, J., Alnajjar, F.S., Gonzalez-Alted, C., Shimoda, S., Pons, J.L., Moreno, J.C.: Haptic Adaptive Feedback to Promote Motor Learning With a Robotic Ankle Exoskeleton Integrated With a Video Game. Frontiers in bioengineering and biotechnology 8 (2020). doi:10.3389/fbioe.2020.00113

160. Kao, P.C., Srivastava, S., Higginson, J.S., Agrawal, S.K., Scholz, J.P.: Short-term Performance-based Error-augmentation versus Error-reduction Robotic Gait Training for Individuals with Chronic Stroke: A Pilot Study. Physical medicine and rehabilitation international 2(9) (2015)

161. Orekhov, G., Fang, Y., Luque, J., Lerner, Z.F.: Ankle Exoskeleton Assistance Can Improve Over-Ground Walking Economy in Individuals With Cerebral Palsy. IEEE Transactions on Neural Systems and Rehabilitation Engineering 28(2), 461-467 (2020)

162. Sulzer, J.S., Roiz, R.A., Peshkin, M.A., Patton, J.L.: A highly backdrivable, lightweight knee actuator for investigating gait in stroke. IEEE Transactions on Robotics 25(3), 539-548 (2009). doi:10.1109/TRO.2009.2019788

163. Lerner, Z.F., Damiano, D.L., Bulea, T.C.: A lower-extremity exoskeleton improves knee extension in children with crouch gait from cerebral palsy. Science Translational Medicine 9(404), 9145 (2017) doi:10.1126/scitranslmed.aam9145

164. Allen, J.L., Kautz, S.A., Neptune, R.R.: Step length asymmetry is representative of compensatory mechanisms used in post-stroke hemiparetic walking. Gait \& posture 33(4), 538-543 (2011)

165. Kerrigan, D.C., Frates, E.P., Rogan, S., Riley, P.O.: Hip hiking and circumduction: quantitative definitions. American journal of physical medicine \& rehabilitation 79(3), 247-252 (2000)

166. Lewek, M.D., Sawicki, G.S.: Trailing limb angle is a surrogate for propulsive limb forces during walking post-stroke. Clinical Biomechanics 67, 115-118 (2019)

167. Buesing, C., Fisch, G., O'Donnell, M., Shahidi, I., Thomas, L., Mummidisetty, C.K., Williams, K.J., Takahashi, H., Rymer, W.Z., Jayaraman, A.: Effects of a wearable exoskeleton stride management

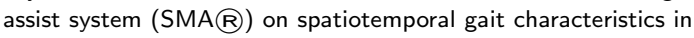
individuals after stroke: A randomized controlled trial. Journal of neuroengineering and rehabilitation 12(1) (2015). doi:10.1186/s12984-015-0062-0

168. Kawamoto, H., Kadone, H., Sakurai, T., Sankai, Y.: Modification of hemiplegic compensatory gait pattern by symmetry-based motion controller of HAL. In: 2015 37th Annual International Conference of the IEEE Engineering in Medicine and Biology Society (EMBC), pp. 4803-4807 (2015)

169. Lee, H.-J., Lee, S.-H., Seo, K., Lee, M., Chang, W.H., Choi, B.-O., Ryu, G.-H., Kim, Y.-H.: Training for walking efficiency with a wearable hip-assist robot in patients with stroke a pilot randomized controlled trial. Stroke 50(12), 3545-3552 (2019) doi:10.1161/STROKEAHA.119.025950

170. Seo, H.G., Lee, W.H., Lee, S.H., Yi, Y., Kim, K.D., Oh, B.-M.: Robotic-assisted gait training combined with transcranial direct current stimulation in chronic stroke patients: A pilot double-blind, randomized controlled trial. Restorative Neurology and Neuroscience 35(5), 527-536 (2017). doi:10.3233/RNN-170745

171. Jung, C., Jung, S., Chun, M.H., Lee, J.M., Park, S., Kim, S.-J.: Development of gait rehabilitation system capable of assisting pelvic movement of normal walking. Acta Medica Okayama 72(4), 407-417 1792 (2018)

172. Duschau-Wicke, A., Von Zitzewitz, J., Caprez, A., Lunenburger, L., Riener, R.: Path control: a method for patient-cooperative robot-aided gait rehabilitation. IEEE Transactions on Neural Systems and Rehabilitation Engineering 18(1), 38-48 (2009)

173. Hidayah, R., Bishop, L., Jin, X., Chamarthy, S., Stein, J., Agrawal, S.K.: Gait Adaptation Using a Cable-Driven Active Leg Exoskeleton (C-ALEX) With Post-Stroke Participants. IEEE Transactions on Neural Systems and Rehabilitation Engineering 28(9), 1984-1993 (2020). doi:10.1109/TNSRE.2020.3009317

174. Bayón, C., Lerma, S., Ramírez, O., Serrano, J.I., Del Castillo, M.D., Raya, R., Belda-Lois, J.M., Martínez, I., Rocon, E.: Locomotor training through a novel robotic platform for gait rehabilitation in pediatric population: short report. Journal of neuroengineering and rehabilitation 13(1), 1-6 (2016). doi:10.1186/s12984-016-0206-x

175. Banala, S.K., Kim, S.H., Agrawal, S.K., Scholz, J.P.: Robot assisted gait training with active leg exoskeleton (ALEX). In: Proceedings of the 2nd Biennial IEEE/RAS-EMBS International Conference on Biomedical Robotics and Biomechatronics, BioRob 2008, pp. 653-658 (2008). doi:10.1109/BIOROB.2008.4762885

176. Wei, D., Li, Z., Wei, Q., Su, H., Song, B., He, W., Li, J.: Human-in-the-Loop Control Strategy of Unilateral Exoskeleton Robots for Gait Rehabilitation. IEEE Transactions on Cognitive and Developmental Systems, 1 (2019)

177. Kaku, A., Parnandi, A., Venkatesan, A., Pandit, N., Schambra, H., Fernandez-Granda, C.: Towards data-driven stroke rehabilitation via wearable sensors and deep learning. arXiv preprint arXiv:2004.08297 (2020)

178. Rupal, B.S., Rafique, S., Singla, A., Singla, E., Isaksson, M., Virk, G.S.: Lower-limb exoskeletons: Research trends and regulatory guidelines in medical and non-medical applications. International Journal of Advanced Robotic Systems 14(6), 1729881417743554 (2017)

179. Vu, H.T.T., Dong, D., Cao, H.-L., Verstraten, T., Lefeber, D., Vanderborght, B., Geeroms, J.: A review of gait phase detection algorithms for lower limb prostheses. Sensors 20(14), 3972 (2020)

180. Bhakta, K., Camargo, J., Donovan, L., Herrin, K., Young, A.: Machine learning model comparisons of user independent \& dependent intent recognition systems for powered prostheses. IEEE Robotics and Automation Letters 5(4), 5393-5400 (2020)

181. Tura, A., Raggi, M., Rocchi, L., Cutti, A.G., Chiari, L.: Gait symmetry and regularity in transfemoral amputees assessed by trunk accelerations. Journal of neuroengineering and rehabilitation $7(1)$, $1-10(2010)$

182. Highsmith, M.J., Schulz, B.W., Hart-Hughes, S., Latlief, G.A., Phillips, S.L.: Differences in the spatiotemporal parameters of transtibial and transfemoral amputee gait. JPO: Journal of Prosthetics and Orthotics 22(1), 26-30 (2010)

183. Vanicek, N., Strike, S., McNaughton, L., Polman, R.: Gait patterns in transtibial amputee fallers vs. non-fallers: Biomechanical differences during level walking. Gait \& Posture 29(3), 415-420 (2009)

184. Fluit, R., Prinsen, E.C., Wang, S., van der Kooij, H.: A comparison of control strategies in commercial and research knee prostheses. IEEE Transactions on Biomedical Engineering 67(1), 277-290 (2019)

185. Tan, X., Zhang, B., Liu, G., Zhao, X., Zhao, Y.: Cadence-insensitive soft exoskeleton design with adaptive gait state detection and iterative force control. IEEE Transactions on Automation Science and Engineering (2021)

186. Park, J.S., Lee, C.M., Koo, S.-M., Kim, C.H.: Gait phase detection using force sensing resistors. IEEE Sensors Journal 20(12), 6516-6523 (2020)

187. Kawamoto, H., Hayashi, T., Sakurai, T., Eguchi, K., Sankai, Y.: Development of single leg version of hal for hemiplegia. In: 2009 Annual International Conference of the IEEE Engineering in Medicine and Biology Society, pp. 5038-5043 (2009). IEEE

188. Calanca, A., Piazza, S., Fiorini, P.: A motor learning oriented, compliant and mobile Gait Orthosis. Applied bionics and biomechanics 9(1), 15-27 (2012). doi:10.1155/2012/123579

189. Bortole, M., Venkatakrishnan, A., Zhu, F., Moreno, J.C., Francisco, G.E., Pons, J.L., Contreras-Vidal, J.L.: The $\mathrm{H} 2$ robotic exoskeleton 
for gait rehabilitation after stroke: Early findings from a clinical study Wearable robotics in clinical testing. Journal of neuroengineering and rehabilitation 12(1) (2015). doi:10.1186/s12984-015-0048-y

190. Kim, S.J., Na, Y., Lee, D.Y., Chang, H., Kim, J.: Pneumatic AFO Powered by a Miniature Custom Compressor for Drop Foot Correction. IEEE Transactions on Neural Systems and Rehabilitation Engineering 28(8), 1781-1789 (2020). doi:10.1109/TNSRE.2020.3003860

191. Nakagawa, K., Tomoi, M., Higashi, K., Utsumi, S., Kawano, R. Tanaka, E., Kurisu, K., Yuge, L.: Short-term effect of a close-fitting type of walking assistive device on spinal cord reciprocal inhibition. Journal of clinical neuroscience : official journal of the Neurosurgical Society of Australasia 77, 142-147 (2020) doi:10.1016/j.jocn.2020.04.121

192. Martínez, A., Durrough, C., Goldfarb, M.: A Single-Joint Implementation of Flow Control: Knee Joint Walking Assistance for Individuals with Mobility Impairment. IEEE Transactions on Neural Systems and Rehabilitation Engineering 28(4), 934-942 (2020). doi:10.1109/TNSRE.2020.2977339

193. Strausser, K.A.: Development of a human machine interface for a wearable exoskeleton for users with spinal cord injury. PhD thesis, UC Berkeley (2011)

194. Ward, J., Sugar, T., Boehler, A., Standeven, J., Engsberg, J.R.: Stroke survivors' gait adaptations to a powered ankle-foot orthosis. Advanced Robotics 25(15), 1879-1901 (2011). doi:10.1163/016918611X588907

195. Arnez-Paniagua, V., Rifaï, H., Amirat, Y., Ghedira, M., Gracies, J.M., Mohammed, S.: Adaptive control of an actuated ankle foot orthosis for paretic patients. Control Engineering Practice 90, 207-220 (2019). doi:10.1016/j.conengprac.2019.06.003

196. Kwon, J., Park, J.-H., Ku, S., Jeong, Y., Paik, N.-J., Park, Y.-L.: A Soft Wearable Robotic Ankle-Foot-Orthosis for Post-Stroke Patients IEEE Robotics and Automation Letters 4(3), 2547-2552 (2019). doi:10.1109/LRA.2019.2908491

197. Yeung, L.-F., Ockenfeld, C., Pang, M.-K., Wai, H.-W., Soo, O.-Y. Li, S.-W., Tong, K.-Y.: Design of an exoskeleton ankle robot for robot-assisted gait training of stroke patients. In: 2017 International Conference on Rehabilitation Robotics (ICORR), pp. 211-215 (2017). IEEE

198. Kim, J.Y., Hwang, S.J., Kim, Y.H.: Development of an active ankle-foot orthosis for hemiplegic patients. In: i-CREATe 2007 Proceedings of the 1st International Convention on Rehabilitation Engineering and Assistive Technology in Conjunction with 1st Tan Tock Seng Hospital Neurorehabilitation Meeting, pp. 110-113 (2007). doi:10.1145/1328491.1328521

199. Forrester, L.W., Roy, A., Hafer-Macko, C., Krebs, H.I., Macko, R.F.: Task-specific ankle robotics gait training after stroke: a randomized pilot study. Journal of neuroengineering and rehabilitation 13(1), 51 (2016). doi:10.1186/s12984-016-0158-1

200. Li, Y., Hashimoto, M.: PVC gel soft actuator-based wearable assist wear for hip joint support during walking. Smart Materials and Structures 26(12) (2017). doi:10.1088/1361-665X/aa9315

201. Swift, T.A., Strausser, K.A., Zoss, A.B., Kazerooni, H.: Control and experimental results for post stroke gait rehabilitation with a prototype mobile medical exoskeleton. In: Dynamic Systems and Control Conference, vol. 44175, pp. 405-411 (2010)

202. Patane, F., Rossi, S., Del Sette, F., Taborri, J., Cappa, P.: WAKE-Up Exoskeleton to Assist Children With Cerebral Palsy: Design and Preliminary Evaluation in Level Walking. IEEE Transactions on Neural Systems and Rehabilitation Engineering 25(7), 906-916 (2017). doi:10.1109/TNSRE.2017.2651404

203. Graf, E.S., Bauer, C.M., Power, V., de Eyto, A., Bottenberg, E., Poliero, T., Sposito, M., Scherly, D., Henke, R., Pauli, C., et al.: Basic functionality of a prototype wearable assistive soft exoskeleton for people with gait impairments: a case study. In: Proceedings of the 11th PErvasive Technologies Related to Assistive Environments Conference, pp. 202-207 (2018)

204. Takahashi, K.Z., Lewek, M.D., Sawicki, G.S.: A neuromechanics-based powered ankle exoskeleton to assist walking post-stroke: A feasibility study. Journal of neuroengineering and rehabilitation 12(1) (2015). doi:10.1186/s12984-015-0015-7
205. Lawrence, S.J., Botte, M.J.: Management of the adult, spastic equinovarus foot deformity. Foot \& ankle international 15(6), 340-346 (1994)

206. Burnfield, M.: Gait analysis: normal and pathological function. Journal of Sports Science and Medicine 9(2), 353 (2010)

207. Sullivan, J.E., Hedman, L.D.: Sensory dysfunction following stroke: incidence, significance, examination, and intervention. Topics in stroke rehabilitation 15(3), 200-217 (2008)

208. O'Sullivan, S.B., Schmitz, T.J. F.A. Davis PT Collection. F. A. Davis Company (1994)

209. Vallery, H., Veneman, J., Van Asseldonk, E., Ekkelenkamp, R., Buss, M., Van Der Kooij, H.: Compliant actuation of rehabilitation robots. IEEE Robotics \& Automation Magazine 15(3), 60-69 (2008)

210. Tariq, M., Trivailo, P.M., Simic, M.: Eeg-based bci control schemes for lower-limb assistive-robots. Frontiers in human neuroscience $\mathbf{1 2}$ 312 (2018)

211. He, Y., Eguren, D., Azorín, J.M., Grossman, R.G., Luu, T.P., Contreras-Vidal, J.L.: Brain-machine interfaces for controlling lower-limb powered robotic systems. Journal of neural engineering 15(2), 021004 (2018)

212. Frolov, A.A., Mokienko, O., Lyukmanov, R., Biryukova, E., Kotov, S., Turbina, L., Nadareyshvily, G., Bushkova, Y.: Post-stroke rehabilitation training with a motor-imagery-based brain-computer interface (bci)-controlled hand exoskeleton: a randomized controlled multicenter trial. Frontiers in neuroscience 11, 400 (2017)

213. López-Larraz, E., Trincado-Alonso, F., Rajasekaran, V. Pérez-Nombela, S., Del-Ama, A.J., Aranda, J., Minguez, J., Gil-Agudo, A., Montesano, L.: Control of an ambulatory exoskeleton with a brain-machine interface for spinal cord injury gait rehabilitation. Frontiers in neuroscience 10, 359 (2016)

214. Balasubramanian, S., Garcia-Cossio, E., Birbaumer, N., Burdet, E., Ramos-Murguialday, A.: Is emg a viable alternative to bci for detecting movement intention in severe stroke? IEEE Transactions on Biomedical Engineering 65(12), 2790-2797 (2018)

215. Maeshima, S., Osawa, A., Nishio, D., Hirano, Y., Takeda, K., Kigawa, H., Sankai, Y.: Efficacy of a hybrid assistive limb in post-stroke hemiplegic patients: a preliminary report. BMC neurology 11(1), 116 (2011)

216. Prasanth, H., Caban, M., Keller, U., Courtine, G., ljspeert, A., Vallery, H., Von Zitzewitz, J.: Wearable sensor-based real-time gait detection: a systematic review. Sensors 21(8), 2727 (2021)

217. Seo, K., Park, Y.J., Lee, J., Hyung, S., Lee, M., Kim, J., Choi, H., Shim, Y.: Rnn-based on-line continuous gait phase estimation from shank-mounted imus to control ankle exoskeletons. In: 2019 IEEE 16th International Conference on Rehabilitation Robotics (ICORR), pp. 809-815 (2019). IEEE

218. Visscher, R.M., Sansgiri, S., Freslier, M., Harlaar, J., Brunner, R., Taylor, W.R., Singh, N.B.: Towards validation and standardization of automatic gait event identification algorithms for use in paediatric pathological populations. Gait \& Posture 86, 64-69 (2021)

219. Yang, S., Zhang, J.-T., Novak, A.C., Brouwer, B., Li, Q.: Estimation of spatio-temporal parameters for post-stroke hemiparetic gait using inertial sensors. Gait \& posture 37(3), 354-358 (2013)

220. Bae, J., Awad, L.N., Long, A., O'Donnell, K., Hendron, K., Holt, K.G., Ellis, T.D., Walsh, C.J.: Biomechanical mechanisms underlying exosuit-induced improvements in walking economy after stroke. The Journal of experimental biology 221(Pt 5) (2018). doi:10.1242/jeb.168815

221. Van Kammen, K., Boonstra, A.M., Van Der Woude, L.H.V., Reinders-Messelink, H.A., Den Otter, R.: Differences in muscle activity and temporal step parameters between Lokomat guided walking and treadmill walking in post-stroke hemiparetic patients and healthy walkers. Journal of neuroengineering and rehabilitation 14(1) (2017). doi:10.1186/s12984-017-0244-z

222. Fleming, A., Stafford, N., Huang, S., Hu, X., Ferris, D.P., Huang, H.H.: Myoelectric control of robotic lower limb prostheses: a review of electromyography interfaces, control paradigms, challenges and future directions. Journal of Neural Engineering (2021)

223. He, Y., Nathan, K., Venkatakrishnan, A., Rovekamp, R., Beck, C. Ozdemir, R., Francisco, G.E., Contreras-Vidal, J.L.: An integrated neuro-robotic interface for stroke rehabilitation using the nasa $\times 1$ 
powered lower limb exoskeleton. In: 2014 36th Annual International Conference of the IEEE Engineering in Medicine and Biology Society, pp. 3985-3988 (2014). IEEE

224. García-Cossio, E., Severens, M., Nienhuis, B., Duysens, J., Desain, P., Keijsers, N., Farquhar, J.: Decoding sensorimotor rhythms during robotic-assisted treadmill walking for brain computer interface (bci) applications. PloS one 10(12), 0137910 (2015)

225. Lapitskaya, N., Nielsen, J.F., Fuglsang-Frederiksen, A.: Robotic gait training in patients with impaired consciousness due to severe traumatic brain injury. Brain injury 25(11), 1070-1079 (2011). doi:10.3109/02699052.2011.607782

226. Esquenazi, A., Lee, S., Wikoff, A., Packel, A., Toczylowski, T., Feeley, J.: A comparison of locomotor therapy interventions: partial-body weight- supported treadmill, lokomat, and g-eo training in people with traumatic brain injury. PM\&R 9(9), 839-846 (2017)

227. Ueba, T., Hamada, O., Ogata, T., Inoue, T., Shiota, E., Sankai, Y.: Feasibility and safety of acute phase rehabilitation after stroke using the hybrid assistive limb robot suit. Neurologia medico-chirurgica 53(5), 287-90 (2013). doi:10.2176/nmc.53.287

228. Borggraefe, I., Schaefer, J.S., Klaiber, M., Dabrowski, E., Ammann-Reiffer, C., Knecht, B., Berweck, S., Heinen, F., Meyer-Heim, A.: Robotic-assisted treadmill therapy improves walking and standing performance in children and adolescents with cerebral palsy. European journal of paediatric neurology 14(6), 496-502 (2010). doi:10.1016/j.ejpn.2010.01.002

229. Wu, M., Kim, J., Arora, P., Gaebler-Spira, D.J., Zhang, Y.: Effects of the Integration of Dynamic Weight Shifting Training into Treadmill Training on Walking Function of Children with Cerebral Palsy: A Randomized Controlled Study. American Journal of Physical Medicine and Rehabilitation 96(11), 765-772 (2017). doi:10.1097/PHM.0000000000000776

230. Weinberger, R., Warken, B., König, H., Vill, K., Gerstl, L., Borggraefe, I., Heinen, F., von Kries, R., Schroeder, A.S.: Three by three weeks of robot-enhanced repetitive gait therapy within a global rehabilitation plan improves gross motor development in children with cerebral palsy - a retrospective cohort study. European journal of paediatric neurology 23(4), 581-588 (2019). doi:10.1016/j.ejpn.2019.05.003

231. Patritti, B.L., Sicari, M., Deming, L.C., Romaguera, F., Pelliccio, M.M., Kasi, P., Benedetti, M.G., Nimec, D.L., Bonato, P.: The role of augmented feedback in pediatric robotic-assisted gait training: A case series. Technology and Disability 22(4), 215-227 (2010). doi:10.3233/TAD-2010-0306

232. Wallard, L., Dietrich, G., Kerlirzin, Y., Bredin, J.: Robotic-assisted gait training improves walking abilities in diplegic children with cerebral palsy. European journal of paediatric neurology : EJPN : official journal of the European Paediatric Neurology Society 21(3), 557-564 (2017). doi:10.1016/j.ejpn.2017.01.012

233. Borggraefe, I., Kiwull, L., Schaefer, J.S., Koerte, I., Blaschek, A., Meyer-Heim, A., Heinen, F.: Sustainability of motor performance after robotic-assisted treadmill therapy in children: an open, non-randomized baseline-treatment study. European Journal of Physical and Rehabilitation Medicine 46(2), 125-131 (2010)

234. Wallard, L., Dietrich, G., Kerlirzin, Y., Bredin, J.: Effect of robotic-assisted gait rehabilitation on dynamic equilibrium control in the gait of children with cerebral palsy. Gait \& posture $60,55-60$ (2018). doi:10.1016/j.gaitpost.2017.11.007

235. Meyer-Heim, A., Ammann-Reiffer, C., Schmartz, A., Schäfer, J., Sennhauser, F.H., Heinen, F., Knecht, B., Dabrowski, E., Borggraefe, I.: Improvement of walking abilities after robotic-assisted locomotion training in children with cerebral palsy. Archives of Disease in Childhood 94(8), 615-620 (2009). doi:10.1136/adc.2008.145458

236. Bayón, C., Martín-Lorenzo, T., Moral-Saiz, B., Ramírez, Ó., Pérez-Somarriba, Á., Lerma-Lara, S., Martínez, I., Rocon, E.: A robot-based gait training therapy for pediatric population with cerebral palsy: Goal setting, proposal and preliminary clinical implementation. Journal of neuroengineering and rehabilitation 15(1) (2018). doi:10.1186/s12984-018-0412-9

237. Forrester, L.W., Roy, A., Krywonis, A., Kehs, G., Krebs, H.I., Macko, R.F.: Modular ankle robotics training in early subacute stroke: a randomized controlled pilot study. Neurorehabilitation and neural repair 28(7), 678-687 (2014). doi:10.1177/1545968314521004

238. Watanabe, H., Marushima, A., Kadone, H., Ueno, T., Shimizu, Y., Kubota, S., Hino, T., Sato, M., Ito, Y., Hayakawa, M., Tsurushima, H., Takada, T., Tsukada, A., Fujimori, H., Sato, N., Maruo, K., Kawamoto, H., Hada, Y., Yamazaki, M., Sankai, Y., Ishikawa, E., Matsumaru, Y., Matsumura, A.: Effects of Gait Treatment With a Single-Leg Hybrid Assistive Limb System After Acute Stroke: A Non-randomized Clinical Trial. Frontiers in Neuroscience 13 (2020). doi:10.3389/fnins.2019.01389

239. Fukuda, H., Samura, K., Hamada, O., Saita, K., Ogata, T., Shiota, E., Sankai, Y., Inoue, T.: Effectiveness of acute phase hybrid assistive limb rehabilitation in stroke patients classified by paralysis severity. Neurologia Medico-Chirurgica 55(6), 487-492 (2015). doi:10.2176/nmc.oa.2014-0431

240. Taki, S., Imura, T., Iwamoto, Y., Imada, N., Tanaka, R., Araki, H., Araki, O.: Effects of Exoskeletal Lower Limb Robot Training on the Activities of Daily Living in Stroke Patients: Retrospective Pre-Post Comparison Using Propensity Score Matched Analysis. Journal of Stroke and Cerebrovascular Diseases 29(10) (2020). doi:10.1016/j.jstrokecerebrovasdis.2020.105176

241. Tan, C.K., Kadone, H., Watanabe, H., Marushima, A., Hada, Y., Yamazaki, M., Sankai, Y., Matsumura, A., Suzuki, K.: Differences in Muscle Synergy Symmetry Between Subacute Post-stroke Patients With Bioelectrically-Controlled Exoskeleton Gait Training and Conventional Gait Training. Frontiers in bioengineering and biotechnology 8, 770 (2020). doi:10.3389/fbioe.2020.00770

242. Watanabe, H., Goto, R., Tanaka, N., Matsumura, A., Yanagi, H.: Effects of gait training using the Hybrid Assistive Limb $\mathbb{R}$ in recovery-phase stroke patients: A 2-month follow-up, randomized, controlled study. NeuroRehabilitation 40(3), 363-367 (2017). doi:10.3233/NRE-161424

243. Yoshikawa, K., Mizukami, M., Kawamoto, H., Sano, A., Koseki, K., Sano, K., Asakawa, Y., Kohno, Y., Nakai, K., Gosho, M., Tsurushima, H.: Gait training with Hybrid Assistive Limb enhances the gait functions in subacute stroke patients: A pilot study. NeuroRehabilitation 40(1), 87-97 (2017). doi:10.3233/NRE-161393

244. Watanabe, H., Tanaka, N., Inuta, T., Saitou, H., Yanagi, H.: Locomotion improvement using a hybrid assistive limb in recovery phase stroke patients: A randomized controlled pilot study. Archives of Physical Medicine and Rehabilitation 95(11), 2006-2012 (2014). doi:10.1016/j.apmr.2014.07.002

245. Kim, S.J., Lee, H.J., Hwang, S.W., Pyo, H., Yang, S.P., Lim, M.-H., Park, G.L., Kim, E.J.: Clinical Characteristics of Proper Robot-Assisted Gait Training Group in Non-ambulatory Subacute Stroke Patients. ANNALS OF REHABILITATION MEDICINE-ARM 40(2), 183-189 (2016). doi:10.5535/arm.2016.40.2.183

246. Goffredo, M., Guanziroli, E., Pournajaf, S., Gaffuri, M., Gasperini, G., 2122 Filoni, S., Baratta, S., Damiani, C., Franceschini, M., Molteni, F., 2123 Befani, S., Cannaviello, G., Colombo, M., Criscuolo, S., De Pisi, F., 2124 Gabbani, D., Galafate, D., Gattini, D., Gison, A., Giovanzana, C., 2125 Giuliani, C., Infantino, D., Infarinato, F., Le Pera, D., Lorenzon, C., 2126 Magoni, L., Marella, R., Marino, M.T., Petruccelli, S., Piermarini, B., 2127 Riolo, S., Riommi, M., Romano, P., Russo, E.F., Russo, M., D'Elia, 2128 T.S., Schiatti, R., Vitullo, V.: Overground Wearable powered 2129 exoskeleton for gait training in subacute stroke subjects: Clinical and 2130 gait assessments. European Journal of Physical and Rehabilitation 2131 Medicine 55(6), 710-721 (2019). doi:10.23736/S1973-9087.19.05574-6

247. Mayr, A., Kofler, M., Quirbach, E., Matzak, H., Fröhlich, K., Prospective, blinded, randomized crossover study of gait 2135 rehabilitation in stroke patients using the Lokomat gait orthosis. 2136 Neurorehabilitation and neural repair 21(4), 307-314 (2007). 2137 doi:10.1177/1545968307300697

248. Cesqui, B., Tropea, P., Micera, S., Krebs, H.I.: Emg-based pattern recognition approach in post stroke robot-aided rehabilitation: a feasibility study. Journal of neuroengineering and rehabilitation 10(1), 1-15 (2013)

249. Lee, S.W., Wilson, K.M., Lock, B.A., Kamper, D.G.: Subject-specific myoelectric pattern classification of functional hand movements for stroke survivors. IEEE Transactions on Neural Systems and Rehabilitation Engineering 19(5), 558-566 (2010) 
250. Geng, Y., Zhang, L., Tang, D., Zhang, X., Li, G.: Pattern recognition based forearm motion classification for patients with chronic hemiparesis. In: 2013 35th Annual International Conference of the IEEE Engineering in Medicine and Biology Society (EMBC), pp. 5918-5921 (2013). IEEE

251. Lu, Z., Tong, K.-y., Zhang, X., Li, S., Zhou, P.: Myoelectric pattern recognition for controlling a robotic hand: a feasibility study in stroke. IEEE Transactions on Biomedical Engineering 66(2), 365-372 (2018)

252. Zhou, H., Zhang, Q., Zhang, M., Shahnewaz, S., Wei, S., Ruan, J., Zhang, X., Zhang, L.: Toward hand pattern recognition in assistive and rehabilitation robotics using emg and kinematics. Frontiers in Neurorobotics 15, 50 (2021)

253. Sczesny-Kaiser, M., Trost, R., Aach, M., Schildhauer, T.A., Schwenkreis, P., Tegenthoff, M.: A Randomized and Controlled Crossover Study Investigating the Improvement of Walking and Posture Functions in Chronic Stroke Patients Using HAL Exoskeleton - The HALESTRO Study (HAL-Exoskeleton STROke Study). Frontiers in Neuroscience 13 (2019). doi:10.3389/fnins.2019.00259

254. Hornby, T.G., Campbell, D.D., Kahn, J.H., Demott, T., Moore, J.L., Roth, H.R.: Enhanced gait-related improvements after therapistversus robotic-assisted locomotor training in subjects with chronic stroke: a randomized controlled study. Stroke 39(6), 1786-1792 (2008). doi:10.1161/STROKEAHA.107.504779

255. Krishnan, C., Kotsapouikis, D., Dhaher, Y.Y., Rymer, W.Z.: Reducing robotic guidance during robot-assisted gait training improves gait function: a case report on a stroke survivor. Archives of physical medicine and rehabilitation 94(6), 1202-1206 (2013). doi:10.1016/j.apmr.2012.11.016

256. Dierick, F., Dehas, M., Isambert, J.-L., Injeyan, S., Bouché, A.-F. Bleyenheuft, Y., Portnoy, S.: Hemorrhagic versus ischemic stroke: Who can best benefit from blended conventional physiotherapy with robotic-assisted gait therapy. PLoS ONE 12(6) (2017) doi:10.1371/journal.pone.0178636

257. Krishnan, C., Ranganathan, R., Kantak, S.S., Dhaher, Y.Y., Rymer, W.Z.: Active robotic training improves locomotor function in a stroke survivor. Journal of neuroengineering and rehabilitation 9, 57 (2012). doi:10.1186/1743-0003-9-57

258. Westlake, K.P., Patten, C.: Pilot study of Lokomat versus manual-assisted treadmill training for locomotor recovery post-stroke. Journal of neuroengineering and rehabilitation 6, 18 (2009). doi:10.1186/1743-0003-6-18

259. Trompetto, C., Marinelli, L., Mori, L., Cossu, E., Zilioli, R., Simonini, M., Abbruzzese, G., Baratto, L.: Postactivation depression changes after robotic-assisted gait training in hemiplegic stroke patients. Gait and Posture 38(4), 729-733 (2013) doi:10.1016/j.gaitpost.2013.03.011

260. Contreras-Vidal, J.L., Bortole, M., Zhu, F., Nathan, K., Venkatakrishnan, A., Francisco, G.E., Soto, R., Pons, J.L.: Neural decoding of robot-assisted gait during rehabilitation after stroke. American journal of physical medicine \& rehabilitation $97(8)$, 541-550 (2018)

261. Wu, M., Landry, J.M., Kim, J., Schmit, B.D., Yen, S.-C., Macdonald, J.: Robotic resistance/assistance training improves locomotor function in individuals poststroke: A randomized controlled study. Archives of Physical Medicine and Rehabilitation 95(5), 799-806 (2014). doi:10.1016/j.apmr.2013.12.021

262. Wu, M., Landry, J.M., Yen, S.-C., Schmit, B.D., Hornby, T.G., Rafferty, M.: A Novel Cable-Driven Robotic Training Improves Locomotor Function in Individuals Post-Stroke. In: 2011 Annual International Conference of the IEEE Engineering in Medicine and Biology Society (EMBC). IEEE Engineering in Medicine and Biology Society Conference Proceedings, pp. 8539-8542 (2011). IEEE; Engn Med \& Biol Soc (EMBS)

263. Yoshimoto, T., Shimizu, I., Hiroi, Y., Kawaki, M., Sato, D., Nagasawa, M.: Feasibility and efficacy of high-speed gait training with a voluntary driven exoskeleton robot for gait and balance dysfunction in patients with chronic stroke: Nonrandomized pilot study with concurrent control. International Journal of Rehabilitation Research 38(4), 338-343 (2015). doi:10.1097/MRR.0000000000000132

264. Kawamoto, H., Kamibayashi, K., Nakata, Y., Yamawaki, K., Ariyasu, R., Sankai, Y., Sakane, M., Eguchi, K., Ochiai, N.: Pilot study of locomotion improvement using hybrid assistive limb in chronic stroke patients. BMC neurology 13, 141 (2013)

doi:10.1186/1471-2377-13-141

265. Yamawaki, K., Ariyasu, R., Kubota, S., Kawamoto, H., Nakata, Y., Kamibayashi, K., Sankai, Y., Eguchi, K., Ochiai, N.: Application of Robot Suit HAL to Gait Rehabilitation of Stroke Patients: A Case Study. In: Miesenberger, $\mathrm{K}$ and Karshmer, $\mathrm{A}$ and Penaz, $\mathrm{P}$ and Zagler, W. (ed.) COMPUTERS HELPING PEOPLE WITH SPECIAL NEEDS, PT II. Lecture Notes in Computer Science, vol. 7383, pp. 184-187 (2012). United Nat Educ, Sci \& Cultural Org; European Disabil Forum; Johannes Kepler Univ Linz

266. Tanaka, H., Nankaku, M., Nishikawa, T., Hosoe, T., Yonezawa, H., Mori, H., Kikuchi, T., Nishi, H., Takagi, Y., Miyamoto, S., Ikeguchi, R., Matsuda, S.: Spatiotemporal gait characteristic changes with gait training using the hybrid assistive limb for chronic stroke patients. Gait \& posture 71, 205-210 (2019). doi:10.1016/j.gaitpost.2019.05.003

267. Bae, Y.-H., Kim, Y.-H., Fong, S.S.M.: Comparison of heart rate reserve-guided and ratings of perceived exertion-guided methods for high-intensity robot-assisted gait training in patients with chronic stroke focused on the motor function and gait ability. Topics in Geriatric Rehabilitation 32(2), 119-126 (2016). doi:10.1097/TGR.0000000000000098

268. Uçar, D.E., Paker, N., Buğdaycı, D.: Lokomat: a therapeutic chance for patients with chronic hemiplegia. NeuroRehabilitation 34(3), 447-453 (2014). doi:10.3233/NRE-141054

269. dos Santos, M.B., de Oliveira, C.B., dos Santos, A., Pires, C.G., Dylewski, V., Arida, R.M.: A Comparative Study of Conventional Physiotherapy versus Robot-Assisted Gait Training Associated to Physiotherapy in Individuals with Ataxia after Stroke. Behavioural neurology 2018 (2018). doi:10.1155/2018/2892065

270. Bae, Y.-H., Lee, S.M., Ko, M.: Comparison of the effects on dynamic balance and aerobic capacity between objective and subjective methods of high-intensity robot-assisted gait training in chronic stroke patients: A randomized controlled trial. Topics in Stroke Rehabilitation 24(4), 309-313 (2017). doi:10.1080/10749357.2016.1275304

271. Bang, D.-H., Shin, W.-S.: Effects of robot-assisted gait training on spatiotemporal gait parameters and balance in patients with chronic stroke: A randomized controlled pilot trial. NeuroRehabilitation 38(4), 343-349 (2016). doi:10.3233/NRE-161325

272. Zhang, J., Fiers, P., Witte, K.A., Jackson, R.W., Poggensee, K.L., Atkeson, C.G., Collins, S.H.: Human-in-the-loop optimization of exoskeleton assistance during walking. Science 356(6344), 1280-1284 (2017)

273. Nuckols, R.W., Sawicki, G.S.: Impact of elastic ankle exoskeleton stiffness on neuromechanics and energetics of human walking across multiple speeds. Journal of neuroengineering and rehabilitation 17(1), 1-19 (2020)

274. Durandau, G., Farina, D., Asin-Prieto, G., Dimbwadyo-Terrer, I., Lerma-Lara, S., Pons, J.L., Moreno, J.C., Sartori, M.: Voluntary control of wearable robotic exoskeletons by patients with paresis via neuromechanical modeling. Journal of neuroengineering and rehabilitation 16 (2019). doi:10.1186/s12984-019-0559-z

275. Durandau, G., Rampeltshammer, W.F., Van Der Kooij, H., Sartori, M.: Myoelectric model-based control of a bi-lateral robotic ankle exoskeleton during even ground locomotion. In: 2020 8th IEEE RAS/EMBS International Conference for Biomedical Robotics and Biomechatronics (BioRob), pp. 822-826 (2020). IEEE

276. Kapeller, A., Felzmann, H., Fosch-Villaronga, E., Hughes, A.-M.: A taxonomy of ethical, legal and social implications of wearable robots: an expert perspective. Science and Engineering Ethics, 1-19 (2020)

277. Hirano, S., Saitoh, E., Tanabe, S., Tanikawa, H., Sasaki, S., Kato, D., Kagaya, H., Itoh, N., Konosu, H.: The features of gait exercise assist robot: precise assist control and enriched feedback. NeuroRehabilitation 41(1), 77-84 (2017) 
Additional Files

Additional file 1 - Analysis of the studies included in the review.

Additional file 2 - Table with the studies included in the clinical

comparison.

2288 Additional file 3 - Relation between outcome metrics and control

2289 strategies for stroke. 


\section{Supplementary Files}

This is a list of supplementary files associated with this preprint. Click to download.

- Additionalfile1.xlsx

- Additionalfile2.xlsx

- Additionalfile3.xlsx 